\begin{tabular}{|c|l|}
\hline Title & Dry minor mergers and size evolution of high-z compact massive early-type gal axies \\
\hline Author(s) & Oogi, Taira; Habe, A sao \\
\hline Citation & $\begin{array}{l}\text { Monthly Notices of the Royal A stronomical Society, 428(1), 641-657 } \\
\text { https://doi.org/10.1093/mnras/sts047 }\end{array}$ \\
\hline Issue Date & 2013-01-01 \\
\hline Doc URL & http://hdl.handle.net/2115/52925 \\
\hline Rights & $\begin{array}{l}\text { This article has been accepted for publication in Monthly Notices of the Royal A stronomical Society @: 2012 The } \\
\text { AuthorsPublished by Oxford University Press on behalf of The Royal A stronomical Society. A Il rights reserved. }\end{array}$ \\
\hline Type & article \\
\hline File Information & MNRA - 2013-0ogi-641-57.pdf \\
\hline
\end{tabular}

Instructions for use 


\title{
Dry minor mergers and size evolution of high- $z$ compact massive early-type galaxies
}

\author{
Taira Oogi ${ }^{\star}$ and Asao Habe ${ }^{\star}$ \\ Department of Cosmosciences, Graduate School of Science, Hokkaido University, Sapporo, Japan
}

Accepted 2012 September 20. Received 2012 September 5; in original form 2011 December 7

\begin{abstract}
Recent observations show evidence that high- $z(z \sim 2-3)$ early-type galaxies (ETGs) are more compact than those with comparable mass at $z \sim 0$. Such size evolution is most likely explained by the 'dry merger sceanario'. However, previous studies based on this scenario cannot consistently explain the properties of both high- $z$ compact massive ETGs and local ETGs. We investigate the effect of multiple sequential dry minor mergers on the size evolution of compact massive ETGs. From an analysis of the Millennium Simulation Data Base, we show that such minor (stellar mass ratio $M_{2} / M_{1}<1 / 4$ ) mergers are extremely common during hierarchical structure formation. We perform $N$-body simulations of sequential minor mergers with parabolic and head-on orbits, including a dark matter component and a stellar component. Typical mass ratios of these minor mergers are $1 / 20<M_{2} / M_{1} \leqq 1 / 10$. We show that sequential minor mergers of compact satellite galaxies are the most efficient at promoting size growth and decreasing the velocity dispersion of compact massive ETGs in our simulations. The change of stellar size and density of the merger remnants is consistent with recent observations. Furthermore, we construct the merger histories of candidates for high- $z$ compact massive ETGs using the Millennium Simulation Data Base and estimate the size growth of the galaxies through the dry minor merger scenario. We can reproduce the mean size growth factor between $z=2$ and $z=0$, assuming the most efficient size growth obtained during sequential minor mergers in our simulations. However, we note that our numerical result is only valid for merger histories with typical mass ratios between $1 / 20$ and $1 / 10$ with parabolic and head-on orbits and that our most efficient size-growth efficiency is likely an upper limit.
\end{abstract}

Key words: methods: numerical - galaxies: elliptical and lenticular, $\mathrm{cD}$ - galaxies: evolution galaxies: formation-galaxies: kinematics and dynamics - galaxies: structure.

\section{INTRODUCTION}

There is significant observational evidence that massive $\left(\gtrsim 10^{11} \mathrm{M}_{\odot}\right)$ early-type galaxies (hereafter ETGs) at high $z(z \sim$ 2-3) are more compact than galaxies with comparable mass in the local Universe. However, a physical explanation for the size evolution from such compact ETGs to local ETGs is still lacking within the $\Lambda \mathrm{CDM}$ galaxy-formation framework.

Many observations of high- $z$ ETGs show that the average size of high- $z$ massive ETGs is smaller by a factor of 3-5 than that of local ETGs with comparable mass (e.g. Trujillo et al. 2006, 2007; Buitrago et al. 2008; Cimatti et al. 2008; van Dokkum et al. 2008). These high- $z$ ETGs also lie below the stellar mass-size relation in

\footnotetext{
^E-mail: oogi@astro1.sci.hokudai.ac.jp (TO); habe@ astro1.sci.hokudai. ac.jp (AH)
}

the local Universe, a relation well investigated through the Sloan Digital Sky Survey (SDSS) data base (e.g. Shen et al. 2003). Additionally, observed high- $z$ ETGs indicate that stellar densities of high- $z$ ETGs are 1-2 orders of magnitude higher than those of local ETGs. Several studies claim that this size growth is even more significant for more massive ETGs (e.g. Newman et al. 2010). Recently, several observations have measured the velocity dispersions of high- $z$ ETGs (e.g. van Dokkum, Kriek \& Franx 2009). The observed velocity dispersions are also higher than the average velocity dispersions of local ETGs with comparable mass (Cappellari et al. 2009; Cenarro \& Trujillo 2009; van Dokkum et al. 2009; van de Sande et al. 2011). Compact massive ETGs at high $z$ are already quiescent in star-formation activity and have an old stellar population (e.g. Cimatti et al. 2008; van Dokkum et al. 2008), similarly to ETGs in the local Universe (e.g. Thomas et al. 2005).

Such compact massive ETGs are extremely rare in the local Universe. Taylor et al. (2010) have searched for compact ETGs in the 
local Universe with the SDSS data base but have only found less massive compact ETGs, which are not readily comparable to the ETGs found at high $z$. Cimatti et al. (2008) also found that such superdense ETGs are extremely rare at $z \approx 0$. On the other hand, Valentinuzzi et al. (2010) claim to find a small number of compact massive ETGs in nearby X-ray-selected clusters and claim that the properties of these galaxies correspond to those of high- $z$ compact massive ETGs. This last example notwithstanding, it is clear that observations indicate that most of the compact massive ETGs at high $z$ must increase their size and decrease their velocity dispersion from $z \simeq 2$ to $z=0$ without recent star formation, in order to keep their old stellar populations. This problem is called 'the size evolution problem of ETGs'.

Dry (i.e. gas-poor, collisionless) mergers are a popular scenario for the size evolution of ETGs. Dry mergers can increase a galaxy's size more than wet (i.e. gas-rich) mergers because of the absence of energy dissipation via the gas component (e.g. Cox et al. 2006; Ciotti, Lanzoni \& Volonteri 2007). Furthermore, dry mergers keep the old stellar populations of progenitor galaxies without the addition of new star formation. There is direct observational evidence for dry mergers (e.g. Bell et al. 2006). Using a semi-analytic approach to galaxy formation, Khochfar \& Silk (2006a) show that massive ETGs experience dry mergers at low redshift $(z<2)$ and suggest that dry mergers are the main driver for the size evolution of ETGs. There are alternative scenarios to dry mergers that involve expansion of the stellar system due to significant mass loss, through either quasars (Fan et al. 2008; Ragone-Figueroa \& Granato 2011) or stellar winds (Damjanov et al. 2009). However, the amount of stellar mass loss is too small to explain the size evolution (Damjanov et al. 2009), compact massive ETGs are already quiescent in star-formation activity and they do not seem to have sufficient gas to puff up the stellar system (Bezanson et al. 2009).

Dry minor mergers are a more effective process for the size evolution of ETGs than dry major mergers. Using the virial theorem, Bezanson et al. (2009) and Naab, Johansson \& Ostriker (2009) make a simple estimate of the size growth for dry mergers. They use initial galaxy models that consist purely of stellar systems and only assume energy conservation during mergers on parabolic orbits. They obtain that, for dry minor mergers, galaxy sizes increase proportional to the square of the mass increase rather than linearly for equal-mass mergers. Semi-analytic models of hierarchical galaxy formation show that minor mergers are an important channel of mass assembly for massive galaxies at $z \lesssim 1-2$ (e.g. Khochfar \& Silk 2006b; Guo $\&$ White 2008). Observational studies suggest that even for massive galaxies the major merger rate is low (e.g. Bundy et al. 2009). It is not likely that major mergers explain the extent of the size evolution of ETGs (Taylor et al. 2010). Therefore, dry minor mergers are likely to be the main driver for the size evolution of massive ETGs.

While a number of numerical simulations have been carried out to study the size evolution problem through dry mergers (e.g. Hopkins et al. 2009; Naab et al. 2009; Nipoti, Treu \& Bolton 2009a; Nipoti et al. 2009b; Hopkins et al. 2010b), they remain insufficient to explain the observed difference between high- $z$ and local ETGs. Several studies have performed high-resolution $N$-body simulations of dry major and minor mergers to investigate the size evolution problem (Nipoti et al. 2009a,b, 2012; Hilz et al. 2012). Nipoti et al. (2009b) simulate a set of dry major and minor mergers and examine the consistency of their results with the stellar-mass scaling relations of local ETGs obtained from the Sloan Lens ACS (SLACS) Survey (Bolton et al. 2008). They conclude that typical local massive ETGs cannot have assembled more than $\sim 45$ per cent of their stellar mass nor grown by more than a factor of $\sim 1.9$ in size via dry mergers. While they did explore the effect of minor mergers, they limited their study to only cases of 'multiple' and 'simultaneous' minor mergers. However, theoretical studies on merger rates indicate that simultaneous minor mergers are extremely rare (e.g. Guo \& White 2008; Stewart et al. 2009; Hopkins et al. 2010a). In the hierarchical structure-formation scenario in the $\Lambda \mathrm{CDM}$ cosmology, 'sequential' minor mergers are more common than simultaneous events.

In this paper, we investigate the effects of dry major and minor mergers on the size evolution of ETGs with $N$-body simulations, focusing particularly on the effects of sequential dry minor mergers and the compactness of the satellite galaxies on the size evolution. To explore the effects of the mergers in the inner regions of compact massive ETGs, we take care to simulate dry minor mergers with sufficient spatial and time resolution. We show that sequential minor mergers of compact satellite galaxies lead to the most efficient size growth and the most efficient decrease of velocity dispersion. Furthermore, we construct the merger histories of candidates of high- $z$ compact massive ETGs using the Millennium Simulation Data Base - a public galaxy catalogue with a semi-analytic model based on the $\Lambda$ CDM cosmology (Springel et al. 2005b; De Lucia $\&$ Blaizot 2007) - and use this to model the size growth of galaxies from dry minor mergers. Based on the size growth efficiency derived by our $N$-body simulations, we discuss the size growth of galaxies from $z \sim 2$ to $z=0$.

This paper is organized as follows. In Section 2, we show the importance of minor mergers for evolution of high- $z$ massive (stellar mass: $M_{*} \geqq 10^{11} \mathrm{M}_{\odot}$ ) galaxies using the Millennium Simulation Data Base. In Section 3, we describe the outline of the simulations, presenting our initial models and choice of simulation parameters in more detail. Results of the analysis of the merger remnants of our simulations are presented in Section 4. In Section 5, we compare our results with observations and discuss the size growth of compact massive ETGs. In Section 6, we give a summary of the paper.

\section{COSMOLOGICAL MERGER HISTORIES OF MASSIVE GALAXIES}

\subsection{Sample selection}

To understand the evolution of ETGs, we examine the massassembly histories of high- $z$ compact massive ETGs. We obtain merging history information for dark matter haloes and those stellar bulges that formed in the centres of dark matter haloes from the results of a semi-analytic model in the Millennium Simulation Data Base (Springel et al. 2005b; De Lucia \& Blaizot 2007). While De Lucia \& Blaizot (2007) use strong supernova and active galactic nucleus (AGN) feedback models to reproduce the observed properties of the brightest cluster galaxies (BCGs), they also adjust their model parameters in order to be in good agreement with observations of the local Universe (see also Croton et al. 2006, using a similar model). Thus, we use this data base as a galaxy-formation model for all galaxies. We extract galaxy samples at $z=0$, the main progenitors of which have a bulge mass more than $10^{11} \mathrm{M}_{\odot}$ at $z=$ 2.07. These galaxies are candidates for high- $z$ compact massive ETGs (van Dokkum et al. 2008). We found 1045 galaxies satisfying this condition in the Millennium Simulation Data Base. In the following subsections, we analyse the merger histories of these sampled galaxies. 


\subsection{The important role of minor mergers} in galaxy mass growth

Fig. 1 shows the number of sample galaxies at different stellar masses taken at $z=0$ (solid lines). These galaxies have experienced major and minor mergers from $z=2.07$ to $z=0$. We define the minor mergers as those mergers with mass ratios $M_{2} / M_{1}<1 / 4$, otherwise we define events as major mergers. We also define 'minor-mergerdominated galaxies' as galaxies for which minor mergers are the dominant form of mass increase during the period $z=2.07$ to $z=$ 0 and we define 'major-merger-dominated galaxies' as galaxies for which major mergers are the dominant effect. In the left panel of Fig. 1, we show the fraction of minor-merger-dominated galaxies in each bulge mass bin, while in the right panel of Fig. 1 we show the fraction in each mass bin for the main dark matter haloes within which the galaxies reside (we call these 'FOF' haloes). We find that $\sim 65$ per cent of the total sample of galaxies are minor-mergerdominated galaxies and $\sim 38$ per cent of the total sample have never experienced major mergers from $z=2.07$ to $z=0$.

In the left panel of Fig. 2, we show the fractional cumulative mass growth of sample galaxies by minor mergers from $z=2.07$ to $z=0$. Here, the fractional cumulative mass growth is the fraction of mass

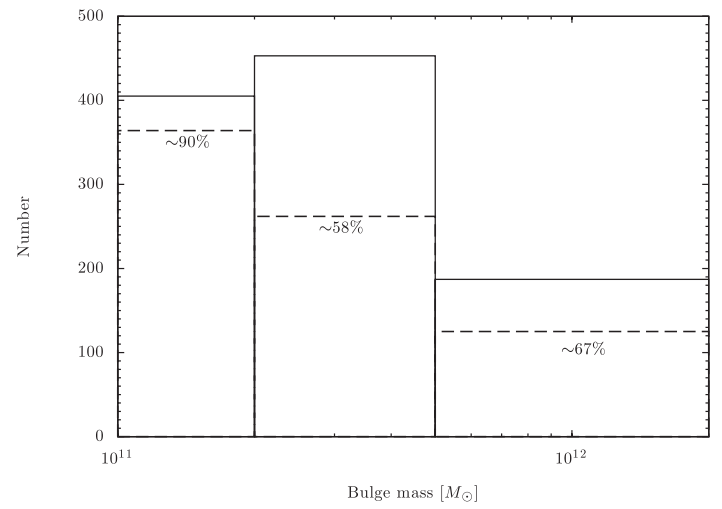

increased by minor mergers from $z=2.07$ to $z=0$ to the bulge mass at $z=0$. We find that on average the fractional cumulative mass growth by minor mergers with mass ratios $M_{2} / M_{1}<1 / 4$ $\left(M_{2} / M_{1}<1 / 10\right)$ is $\sim 0.3(\sim 0.2)$ and more massive galaxies have larger fractional cumulative mass growth through minor mergers. Thus, minor mergers are one of the dominant processes for mass growth of our sample galaxies. In the right panel of Fig. 2, we show the dependence of the fractional cumulative mass growth on the mass of FOF haloes. We find that many galaxies have increased their mass significantly by minor mergers in more massive FOF haloes. Therefore, we expect that these minor mergers increase galaxy sizes, in particular in high-density environments. ETGs are likely to be in such high-density environments, a fact well-known as the morphology-density relation (Dressler 1980). For this reason, in this paper we concentrate on minor mergers as the one of the important processes driving the size evolution of ETGs.

\subsection{Stellar merger histories of sample galaxies}

We analyse the merging events of the 1045 sample galaxies and show that minor merger events are sequential processes rather than

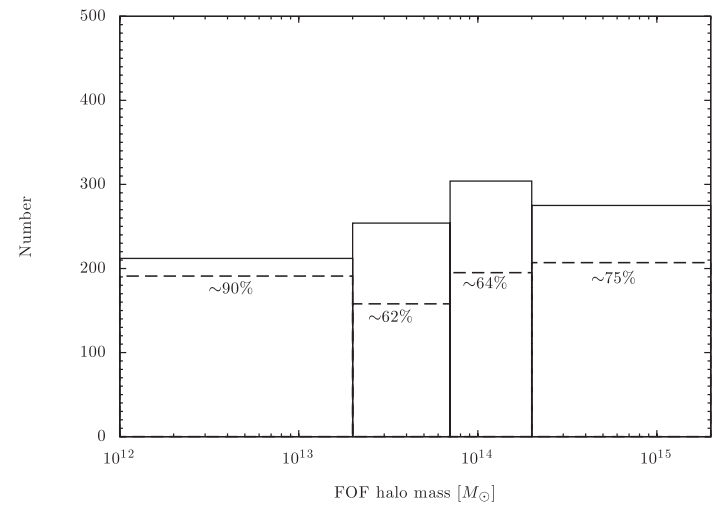

Figure 1. Left: number of sample galaxies derived by the Millennium Simulation Data Base (solid lines). The horizontal axis shows the bulge mass of the galaxies at $z=0$, while the vertical axis shows the number of galaxies in each mass bin. The mass bins are $\left[1.0 \times 10^{11}: 2.0 \times 10^{11}\right],\left[2.0 \times 10^{11}: 5.0 \times 10^{11}\right]$ and $\left[5.0 \times 10^{11}: 2.0 \times 10^{12}\right]$ in units of $\mathrm{M}_{\odot}$. Fractions of minor-merger-dominated galaxies (see text) are also plotted (dashed lines with percentages). Right: the same as the left panel, but with the horizontal axis showing the FOF halo mass of the galaxies at $z=0$. The mass bins are $\left[1.0 \times 10^{12}: 2.0 \times 10^{13}\right]$, $[2.0 \times$ $\left.10^{13}: 7.0 \times 10^{13}\right],\left[7.0 \times 10^{13}: 2.0 \times 10^{14}\right]$ and $\left[2.0 \times 10^{14}: 2.0 \times 10^{15}\right]$ in units of $\mathrm{M}_{\odot}$.
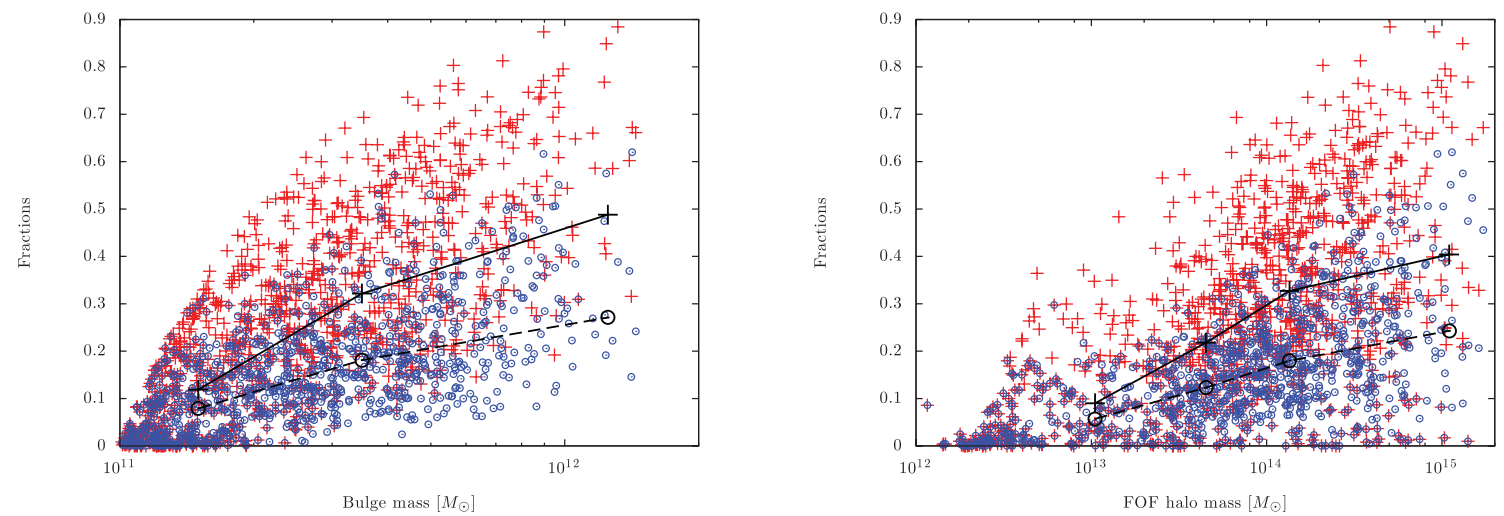

Figure 2. Left: fractional cumulative mass growth through minor mergers with mass ratios $M_{2} / M_{1}<1 / 4$ and $M_{2} / M_{1}<1 / 10$ from $z=2.07$ to $z=0$ for our sample galaxies derived by the Millennium Simulation Data Base. Here, the fractional cumulative mass growth is the fraction of mass increased by minor mergers from $z=2.07$ to $z=0$ to the mass of the bulge at $z=0$. The horizontal axis shows the bulge mass at $z=0$. Coloured crosses (red in the online article) represent the fractional cumulative mass growth with mass ratios $M_{2} / M_{1}<1 / 4$. We average these in the same mass bins as Fig. 1 and show the average values with black crosses and the solid line. Coloured open circles (blue in the online article) represent mass ratios $M_{2} / M_{1}<1 / 10$. We also average these and show the averages with black open circles and the dashed line. Right: the same as the left panel, but for a horizontal axis showing the FOF halo mass of the galaxies at $z=0$. 

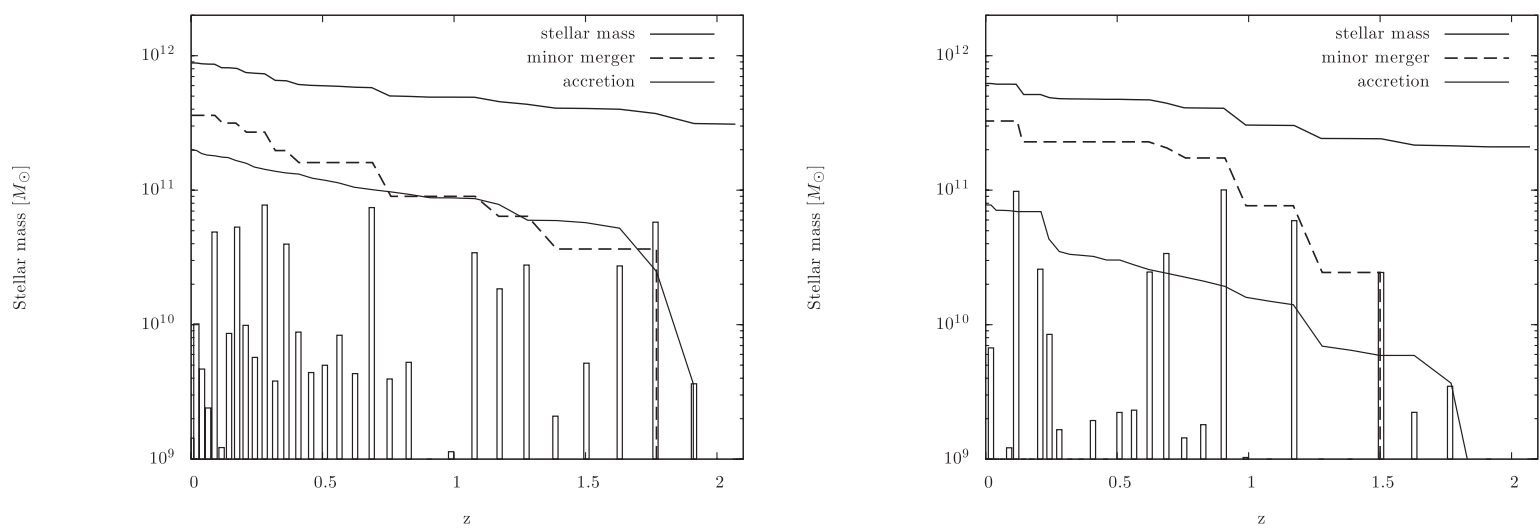

Figure 3. Stellar merger histories of our two sample galaxies derived by the Millennium Simulation Data base. These have never experienced major mergers. Left: a central galaxy of a FOF halo with mass $1.5 \times 10^{15} \mathrm{M}_{\odot}$. Right: a member galaxy of a FOF halo with mass $1.8 \times 10^{14} \mathrm{M}_{\odot}$. The thick solid line is the stellar mass of the galaxies as a function of redshift. The bars in each panel show the increased mass by minor mergers at each event. Dashed and dotted lines show the cumulative increased stellar mass from $z=2.07$ to $z=0$ from minor mergers and accretions, respectively. We define accretions as mergers with mass ratios less than 1:20.

simultaneous events between $z=2.07$ and $z=0$. We obtain the merging epochs at which a satellite (less massive) galaxy merges into the primary (more massive) galaxy from the results of the semianalytic model (see Croton et al. 2006). We identify 19605 minor mergers, where we count multiple minor mergers as one minor merging event if they occur during a time interval of $\sim 0.35 \mathrm{Gyr}$, corresponding to the dynamical time-scale of the dark matter haloes of the galaxies at $z \sim 2$. Except for one case, the sum of the mass accreted through minor mergers during this time interval is less than 50 per cent of the mass of the main progenitor galaxy. Therefore, almost all minor mergers occur sequentially.

Fig. 3 shows the stellar merger histories of two sample galaxies. One is a central galaxy of a FOF halo, the mass of which is $1.5 \times$ $10^{15} \mathrm{M}_{\odot}$, and another is a member galaxy of a FOF halo, the mass of which is $1.8 \times 10^{14} \mathrm{M}_{\odot}$. These two galaxies have experienced sequential minor mergers but have not experienced major mergers since $z=2.07$. We find that the total merged mass through minor mergers is comparable to the stellar mass at $z=2.07$. Therefore, the size evolution is driven significantly by this process. In this paper, we focus on such 'sequential' minor merging processes.

\section{$3 N$-BODY SIMULATIONS}

In this section, we describe our initial galaxy models, model parameters, $N$-body simulation methods and methods of analysing merger remnants.

\subsection{Initial galaxy models}

Our model galaxies consist of two components: a stellar bulge and a dark matter halo. We model the dark matter mass distribution with a Hernquist (1990) profile that has total mass $M_{\mathrm{dm}}$ :

$\rho_{\mathrm{dm}}(r)=\frac{M_{\mathrm{dm}}}{2 \pi} \frac{a_{\mathrm{dm}}}{r\left(r+a_{\mathrm{dm}}\right)^{3}}$,

scaled to match the Navarro-Frenk-White (NFW) profile found in cosmological simulations (Navarro, Frenk \& White 1997) as described in Springel, Di Matteo \& Hernquist (2005a). In this model, the total mass, $M_{\mathrm{dm}}$, corresponds to the mass within the virial radius of the NFW model and the inner density profile of the Hernquist profile is also equal to that of the NFW model. Satisfying the above condition, the scale radius of the Hernquist profile $a_{\mathrm{dm}}$ is related to the scalelength $r_{\mathrm{s}}$ of the NFW halo and halo concentration $c_{\mathrm{vir}}\left(c_{\mathrm{vir}}=\right.$ $\left.r_{\mathrm{vir}} / r_{\mathrm{s}}\right)$ as

$a_{\mathrm{dm}}=r_{\mathrm{s}} \sqrt{2\left[\ln \left(1+c_{\mathrm{vir}}\right)-c_{\mathrm{vir}} /\left(1+c_{\mathrm{vir}}\right)\right]}$.

We derive the virial radius of the halo from a spherical collapse model, $r_{\mathrm{vir}}=r_{\mathrm{vir}}\left(M_{\mathrm{dm}}, z\right)$ (e.g. Barkana \& Loeb 2001), where $z$ is the formation redshift of the halo. Using these parameters of mass, redshift and halo concentration for a dark matter halo, we can obtain the corresponding halo model for an $\mathrm{N}$-body realization.

We also model the initial distribution of the bulge with a Hernquist profile:

$\rho_{*}(r)=\frac{M_{*}}{2 \pi} \frac{a}{r(r+a)^{3}}$,

with total stellar mass $M_{*}$, quarter-mass radius $r_{1 / 4}=a$ and halfmass radius $r_{1 / 2}=(1+\sqrt{2}) a$. Observationally, a galaxy size is usually characterized by its effective radius, defined as the radius of the isophote that encloses half of the total stellar luminosity in projection. In our simulations, we adopt the effective radius $R_{\mathrm{e}}$ as the projected radius enclosing half of the stellar mass. Here we assume a constant stellar mass-to-light ratio. From equation (3), the effective radius is related to the quarter- and half-mass radii by $R_{\mathrm{e}}=$ $1.8153 r_{1 / 4}=0.752 r_{1 / 2}$ (see also Binney \& Merrifield 1998). We remove particles of the two-component Hernquist profile outside $r \simeq$ $20 a_{\mathrm{dm}}$ in our numerical realizations in order to avoid the occurrence of a very small number of very distant particles. This choice of outer radius does not affect the half-mass radius significantly. If the total stellar mass and the scale radius are specified, the Hernquist profile determines a unique structure.

We construct the bulge and the dark matter halo to be in equilibrium in the total gravitational potential by an $\mathrm{N}$-body method. We assume that both components are both spherical and isotropic. The particle positions for each component are initialized from the density profile, while the particle velocities are drawn based on each component's equilibrium distribution function $f_{i}(E)$ (Binney $\&$ Tremaine 2008):

$f_{i}(E)=\frac{1}{\sqrt{8} \pi^{2}} \int_{0}^{E} \frac{\mathrm{d}^{2} \rho_{i}}{\mathrm{~d} \psi^{2}} \frac{\mathrm{d} \psi}{\sqrt{E-\psi}}$,

where $E$ is the relative energy of each particle of component $i, \rho_{i}$ is the density profile of component $i$ and $\psi$ is the total gravitational potential. Ciotti (1996) derives the analytical distribution function 
Table 1. Initial galaxy models for dry merger simulations. First column: name of the initial galaxy model. Model A is a compact massive ETG seen at high $z$. This is the primary galaxy in our minor merger simulations. Model B is a compact, less massive ETG. This is used as a compact satellite. Model C is a less massive ETG that obeys the stellar mass-size relation in the local Universe. Model D is the most compact satellite (see Section 3.2). Columns 2-10: dark matter halo mass; redshift; halo concentration; virial radius of the halo measured at each redshift; number of dark matter particles; stellar mass; effective radius; number of stellar particles; softening length.

\begin{tabular}{cccccccccc}
\hline Model & $M_{\mathrm{dm}}\left(\mathrm{M}_{\odot}\right)$ & $z$ & $c$ & $r_{\text {vir }}(\mathrm{kpc})$ & $N_{\mathrm{dm}}$ & $M_{*}\left(\mathrm{M}_{\odot}\right)$ & $R_{\mathrm{e}}(\mathrm{kpc})$ & $N_{*}$ & $\epsilon(\mathrm{kpc})$ \\
\hline A & $10^{12}$ & 2.0 & 10 & 105 & $6.0 \times 10^{5}$ & $10^{11}$ & 1.0 & $6.0 \times 10^{4}$ & 0.03 \\
B & $10^{11}$ & 2.0 & 10 & 48.8 & $6.0 \times 10^{4}$ & $10^{10}$ & 0.464 & $6.0 \times 10^{3}$ & 0.03 \\
C & $10^{11}$ & 0.0 & 10 & 120 & $6.0 \times 10^{4}$ & $10^{10}$ & 1.15 & $6.0 \times 10^{3}$ & 0.03 \\
D & $10^{11}$ & 2.0 & 10 & 48.8 & $6.0 \times 10^{4}$ & $10^{10}$ & 0.148 & $6.0 \times 10^{3}$ & 0.03 \\
\hline
\end{tabular}

of the two-component Hernquist profiles. We use this formula to obtain the particle velocity distribution.

Here, we only briefly summarize our $N$-body realization method, which is described in detail in Kuijken \& Dubinski (1994). A particle's position is first determined by sampling from the density distribution. Next the particle's velocity vector is randomly sampled from inside a velocity-space sphere, the radius of which is the local escape velocity, $v_{\text {esc }}=\sqrt{-2 \psi}$. Finally, based on the distribution function of the bulge or halo component, we determine whether we select the velocity vector or not using the acceptance-rejection technique.

\subsection{Model parameters}

For the progenitor galaxies in major and minor merger simulations, we assume four galaxy models: a massive ETG, two less massive compact ETGs and a less massive diffuse ETG. Parameters of these galaxy models are summarized in Table 1 . We assume that the massive ETG (model A) has stellar mass $M_{*}=10^{11} \mathrm{M}_{\odot}$, with an effective radius $R_{\mathrm{e}}=1.0 \mathrm{kpc}$, which corresponds to the scale radius $a=0.551 \mathrm{kpc}$. These properties are consistent with those from observations of high- $z$ compact massive ETGs (e.g. Trujillo et al. 2007). They are embedded in dark matter haloes of $M_{\mathrm{dm}}=$ $10^{12} \mathrm{M}_{\odot}$ and $r_{\text {vir }}$ at $z=2.0$. In this paper, we assume a dark matter to stellar mass ratio $M_{\mathrm{dm}} / M_{*}=10$ in all models. We also assume that for all initial dark matter haloes, $c_{\mathrm{vir}}=10$. Many cosmological simulations predict that the halo concentration depends on the mass and the formation redshift (Bullock et al. 2001) but we select a constant value in favour of simplicity in the model.

We assume three different satellite galaxies: compact satellites (model B), diffuse satellites (model C) and very compact satellites (model D). We assume that the formation epoch of model B is the same as that of model A, both occurring at $z=2.0$, and that these models are expected to have the same average density. Based on this assumption, we construct the stellar system for model B by simply scaling down that of model $A$, given the mass fraction (1/10 of model A) and scale radius $(1 / \sqrt[3]{10}$ of model A). We also assume that the dark matter halo of model B is $M_{\mathrm{dm}}=10^{11} \mathrm{M}_{\odot}$. For model $\mathrm{C}$ galaxies, we assume that they have the properties of local ETGs. For their effective radii, we use the observed local stellar mass-size relation of early-type galaxies in the SDSS (Shen et al. 2003):

$R_{\mathrm{e}}=4.16\left(\frac{M_{*}}{10^{11} \mathrm{M}_{\odot}}\right)^{0.56} \mathrm{kpc}$.

The model $C$ galaxies have dark matter haloes with $M_{\mathrm{dm}}=10^{11} \mathrm{M}_{\odot}$ and $r_{\text {vir }}$ at $z=2.0$. Recent observations indicate that early-type galaxies follow a stellar mass-size relation $\log \left(R_{\mathrm{e}}\right)=S+T \log \left(M_{*}\right)$ with $T \sim 0.6-0.7$, not significantly dependent on redshift, and $S$ increasing for decreasing redshift (e.g. Newman et al. 2012). From this, we assume that model A and model D lie on the same stellar mass-size relation with $T=0.57$, as in Newman et al. (2012), giving model D satellites that are more compact than both models B and C. We examine the effects of the stellar densities of the satellites on the properties of the merger remnants using this set of satellite models.

\subsection{Simulations and test run}

We use the GADGET- 2 code (Springel 2005), an efficient parallelized treecode, to perform our simulations. We set the tolerance parameter $\alpha=0.001$ for the relative cell-opening criterion in the tree algorithm, the time-step tolerance parameter $\eta=0.005$ and the force-softening $\epsilon=0.03 \mathrm{kpc}$ for both star and dark matter particles. With this choice, the minimum time-step is $<0.02 \mathrm{Myr}$. In this study, we use $6.0 \times 10^{5}$ dark matter particles and $6.0 \times 10^{4}$ stellar particles for model A. For models B and C, we use $6.0 \times 10^{4}$ dark matter particles and $6.0 \times 10^{3}$ stellar particles. Each halo and stellar particle has the same mass.

We should draw attention to the effects of two-body relaxation, since the dynamical time of compact massive ETGs is relatively short compared with the galaxy merging time-scale and we need the simulation time to be much larger than the dynamical time of the stellar system. We have confirmed that our two-component galaxy model is stable at all radii $r>a=0.551 \mathrm{kpc}$ for $\sim 3.6 \mathrm{Gyr}$ of the simulation. We define the half-mass dynamical time of a stellar system as

$t_{\mathrm{dyn}}=\sqrt{\frac{3 \pi}{16 G \rho_{1 / 2}}}=\pi \sqrt{\frac{r_{1 / 2}^{3}}{2 G M_{*}}}$,

where $\rho_{1 / 2}=3 M_{*} / 8 \pi r_{1 / 2}^{3}$ is the mean density inside the half-mass radius $r_{1 / 2}$. Here, we consider only the stellar component for simplicity. We estimate the two-body relaxation time-scale $t_{\mathrm{r}}$ from the following expression:

$t_{\mathrm{r}}(r) \simeq \frac{0.1 N}{\ln N} t_{\mathrm{dyn}}$,

where $N$ is the number of stellar particles within a radius $r$. The halfmass dynamical time of model $\mathrm{A}$ is $t_{\mathrm{dyn}} \simeq 5.2 \mathrm{Myr}$ and the relaxation time is $t_{\mathrm{r}} \simeq 2 \mathrm{Gyr}$, which is comparable with the total computational time of each of our simulation sets. To check whether our twocomponent galaxy model is stable, we simulated a single model A galaxy for $\sim 3.6 \mathrm{Gyr}$ as a test run. Fig. 4 shows several snapshots of the density profiles of the stellar system. This figure clearly shows that our model is stable at all radii $r \gtrsim 0.2 \mathrm{kpc}$. We checked the conservation of total energy $E$ and the virial ratio $(V=2 T / W)$, obtaining $|\Delta E / E| \lesssim 1$ per cent and $|\Delta V / V| \lesssim 0.35$ per cent. We also checked the stellar half-mass radius, the stellar effective radius and the stellar velocity dispersion at $3.6 \mathrm{Gyr}$ and we confirmed that 


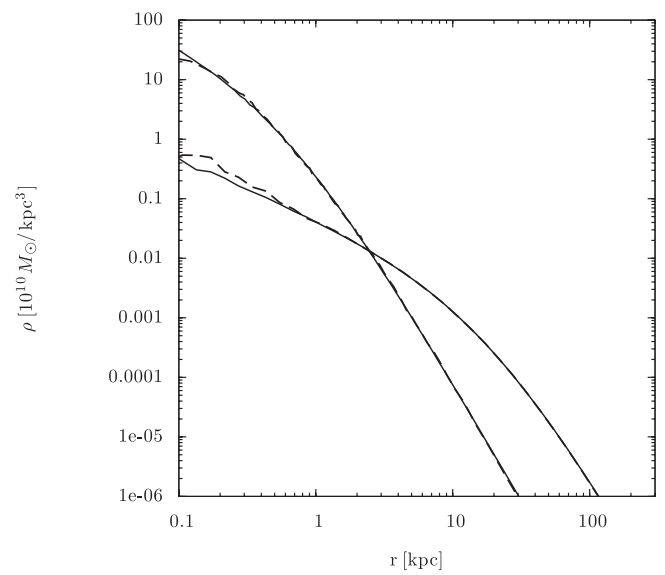

Figure 4. Density profiles of the stellar system and dark matter halo of a single model A galaxy (Table 1) simulated as a test run to check whether our two-component galaxy model is stable. The solid line shows the initial profile $(t=0)$. The dashed line shows the profile after $3.6 \mathrm{Gyr}$. The more concentrated profiles represent the stellar system.

changes in both the half-mass radius and the effective radius are less than 3 per cent and that the projected velocity dispersion is nearly constant. These changes are smaller than those due to mergers in all runs (see Table 2). In order to avoid the influence of numerical error, we stopped our simulations at 3.6 Gyr.

\subsection{Production run set}

We have run $\mathrm{N}$-body simulations of dry major and minor mergers to investigate the effects of mergers on the evolution of size and velocity dispersion in ETGs. In this paper, we mainly focus on the dry minor merger cases. We also simulate a dry major merger for comparison. We describe the set-up of our simulations below. Table 2 summarizes the parameters used in our run set.

Merger orbits are critical to the properties of merger remnants. We assume head-on parabolic mergers with zero orbital angular momentum in all our simulations. In the work of Boylan-Kolchin,
Ma \& Quataert (2006), who studied dry major mergers of earlytype galaxies, merger-remnant properties are highly influenced by the orbital parameters of the mergers. They showed that the stellar bulges of the merger remnants in the case of radial orbits are larger than in the case of circular orbits. Therefore, our simulations are optimal cases for the size growth of galaxies.

\subsubsection{Major mergers}

In run $2 \mathrm{~A}$, we simulate a major merger for a parabolic and headon encounter of the same initial galaxies as in model A. The initial separation between the two galaxies is $200 \mathrm{kpc}$ and the initial relative speed is $308 \mathrm{~km} \mathrm{~s}^{-1}$. The merger appears to be mostly completed by $2.3 \mathrm{Gyr}$.

\subsubsection{Minor mergers}

We simulate two cases of minor mergers, involving simultaneous minor mergers and sequential ones. We investigate how merger remnants are affected by different types of minor mergers. In each case, we assume three different model galaxies (models B, C and $\mathrm{D}$, as in Table 1). Initially, we assume that the central galaxy of model A (the 'primary galaxy') is surrounded by satellite galaxies of model type B, C or D. In the simultaneous minor mergers case, the satellites are randomly distributed around the primary galaxy with relative distance between each satellite and the primary galaxy in the range $[130,170] \mathrm{kpc}$. During the merging events, the satellites are accreted at the same time. The merger orbit of each satellite is assumed to be parabolic and head-on in the absence of the other satellites. We calculate the relative velocity using the total mass of the progenitors before removing very distant particles.

As shown in Section 2, in the hierarchical structure-formation scenario in the $\Lambda \mathrm{CDM}$ cosmology there are many sequential minor mergers. We study the effects of this sequential minor merging in our next case. Here, each satellite merges with the primary galaxy from a random direction every $0.2 \mathrm{Gyr}$. We simulate five sequential (runs 1A5Bsq, 1A5Csq, 1A5Dsq) and then 10 sequential (runs 1A10Bsq, 1A10Csq, 1A10Dsq) minor mergers. In these cases, the period

Table 2. Physical properties of the merger remnants within the fixed radius $60 \mathrm{kpc}$ in each run. The properties of the initial galaxy are defined within $60 \mathrm{kpc}$. First column: name of the simulation. 2A: major merger of model A; $1 \mathrm{~A}$ : the primary galaxy; 1/5/10: the number of minor mergers; B/C/D: type of the satellite galaxy; sq/sm: sequential or simultaneous minor mergers. Columns 2-10: stellar mass; virial radius of the dark matter halo; half-mass radius of the stellar system; effective radius; average density within $1 \mathrm{kpc}$; average density within the effective radius; velocity dispersion; size growth efficiency; efficiency of the change of velocity dispersion (see Section 4.5).

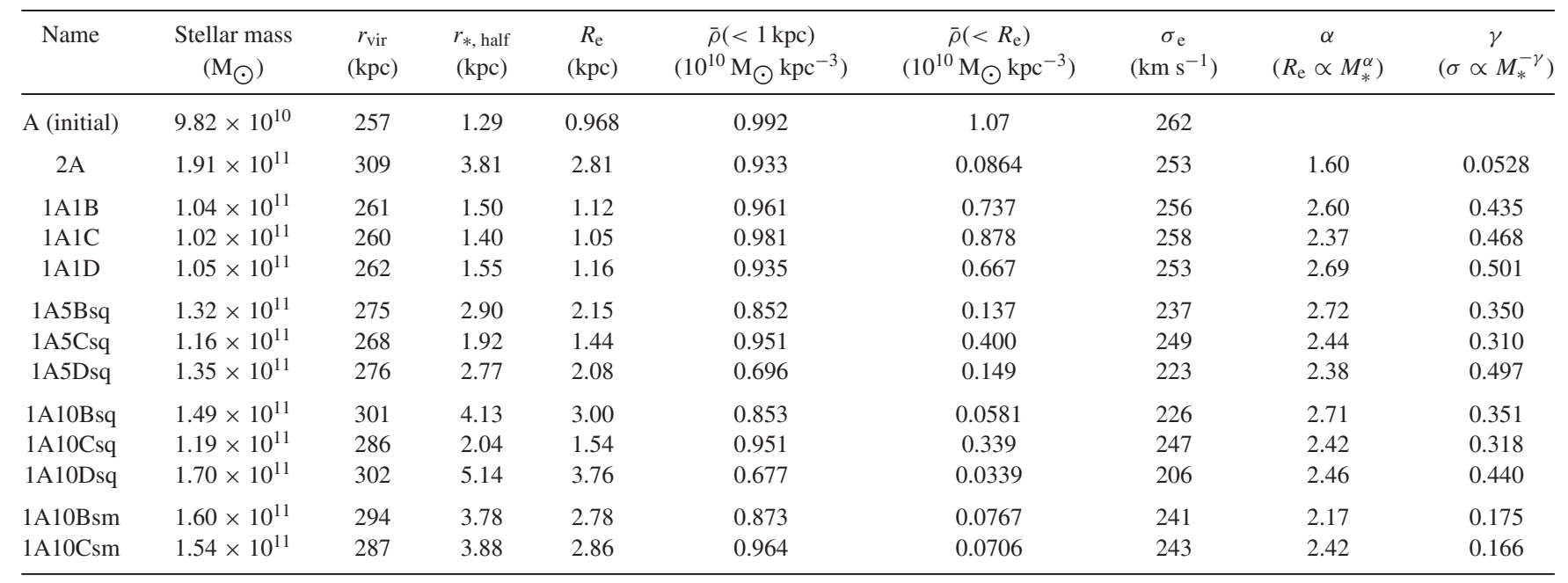


Table 3. Physical properties of the merger remnants within $r_{\text {trunc }}=30,60$ and $90 \mathrm{kpc}$ in binary minor merger simulations. Each column is similar to Table 2.

\begin{tabular}{cccccc}
\hline Name & $\begin{array}{c}\text { Stellar mass } \\
\left(\mathrm{M}_{\odot}\right)\end{array}$ & $\begin{array}{c}R_{\mathrm{e}} \\
(\mathrm{kpc})\end{array}$ & $\begin{array}{c}\sigma_{\mathrm{e}} \\
\left(\mathrm{km} \mathrm{s}^{-1}\right)\end{array}$ & $\begin{array}{c}\alpha \\
\left(R_{\mathrm{e}} \propto M_{*}^{\alpha}\right)\end{array}$ & $\begin{array}{c}\gamma \\
\left(\sigma \propto M_{*}^{-\gamma}\right)\end{array}$ \\
\hline$r_{\text {ana }}=30 \mathrm{kpc}$ & & & & & \\
1A1B & $1.01 \times 10^{11}$ & 1.06 & 257 & 2.71 & 0.535 \\
1A1C & $0.984 \times 10^{11}$ & 0.990 & 259 & 2.63 & 0.758 \\
1A1D & $1.02 \times 10^{11}$ & 1.11 & 254 & 2.79 & 0.569 \\
$r_{\text {ana }}=60 \mathrm{kpc}$ & & & & & \\
1A1B & $1.04 \times 10^{11}$ & 1.12 & 256 & 2.60 & 0.435 \\
1A1C & $1.02 \times 10^{11}$ & 1.05 & 258 & 2.37 & 0.468 \\
1A1D & $1.05 \times 10^{11}$ & 1.16 & 253 & 2.69 & 0.501 \\
$r_{\text {ana }}=90 \mathrm{kpc}$ & & & & & \\
1A1B & $1.05 \times 10^{11}$ & 1.14 & 256 & 2.55 & 0.408 \\
1A1C & $1.03 \times 10^{11}$ & 1.07 & 257 & 2.28 & 0.457 \\
1A1D & $1.06 \times 10^{11}$ & 1.18 & 252 & 2.67 & 0.534 \\
\hline
\end{tabular}

of the sequential minor mergers roughly corresponds to the time interval from $z=2$ to $z=1$. We assume the three types of satellites (models B, C and D) and compare the properties of the merger remnants. In the sequential minor mergers, the mass ratio of the first merger is $M_{2} / M_{1}=1 / 10$, that of the second merger is $1 / 11$, and so on. The mass ratio can reach $\sim 1 / 20$ in the last merger. Thus, the typical mass ratios of minor mergers are $1 / 20<M_{2} / M_{1} \leqq 1 / 10$. We also simulate single minor mergers (runs 1A1B, 1A1C, 1A1D) to compare these results with sequential minor mergers. In these test simulations, we set the relative velocity of the satellite using the total mass of the progenitors after we have removed very distant particles to ensure that the satellite orbits are strictly parabolic and head-on.

Numerical simulations were run on Cray XT4 at the Center for Computational Astrophysics (CfCA) of the National Astronomical Observatory of Japan.

\subsection{Definition of merger remnants}

In this subsection, we describe our analysis methods for the merger remnants. In the major merger simulation (run $2 \mathrm{~A}$ ), the final state is defined when the global virial ratio of the system reaches an almost constant value. In the case of the minor mergers, it takes more time for the mergers to attain complete relaxation, since the dynamical friction time-scale of the minor mergers is longer than the merging time-scale of the major merger. Therefore, as described in Section 3.3, we stop our runs at $t=3.6 \mathrm{Gyr}$ in the minor merger simulations and analyse the merger remnants. At this time, there are several surviving cores of satellite stellar systems in the remnants from the 10 minor mergers. However, the mass of each core is less than 5 per cent of the mass of the merger remnant, which we define below. We examine the effects of these surviving cores on the properties of merger remnants in Section 4.3 and find that these cores have little effect on the size growth efficiency (defined in Section 4.5) of the remnants.

We analyse the remnant particles at the end of the simulations. For dark matter particles, we define the merger remnants as those particles that are gravitationally bound. For stellar particles it is difficult to use that criterion, since in the minor merger simulations the central galaxies are surrounded by very diffuse stellar particles beyond $\gtrsim 50 \mathrm{kpc}$ that are gravitationally bound by the dark matter haloes of the remnants. Since van Dokkum et al. (2010) use $75 \mathrm{kpc}$ as the robust galaxy radius to define total stellar mass in their stacked images and determine the effective radii, we use a radius $r_{\text {trunc }}=$ $60 \mathrm{kpc}$ to define the stellar remnants, with our reasoning described below. We call the stellar mass within $r_{\text {trunc }}$ the remnant mass. At $r>r_{\text {trunc }}$, the stellar surface density of the remnants is too low to be observed. In fact, the surface brightness difference between 0.1 and $60 \mathrm{kpc}$ is $\Delta \mu \gtrsim 13 \mathrm{mag} \operatorname{arcsec}^{-2}$ for the remnants, assuming a constant mass-to-light ratio with radius. Even state-of-theart observations cannot observe such a large range of magnitudes (Trujillo, Carrasco \& Ferre-Mateu 2012). We examine various radii $r_{\text {trunc }}$ to find a suitable $r_{\text {trunc }}$ by which to define the remnants. We have checked $R_{\mathrm{e}}$ for $r_{\text {trunc }}=30,60$ and $90 \mathrm{kpc}$ in the binary minor merger simulations, as shown in Table 3 . We find that $R_{\mathrm{e}}$ slightly increases from $r_{\text {trunc }}=30 \mathrm{kpc}$ to $r_{\text {trunc }}=60 \mathrm{kpc}$ and is almost constant between $r_{\text {trunc }}=60 \mathrm{kpc}$ and $r_{\text {trunc }}=90 \mathrm{kpc}$. Thus, we choose $r_{\text {trunc }}=60 \mathrm{kpc}$ in the following analysis of merger remnants.

We analyse the three-dimensional and projected properties of the stellar merger remnants. The stellar half-mass radius is the radius of the sphere within which half of the mass of the remnant stellar system is enclosed. We adopt the effective radius $R_{\mathrm{e}}$ as the projected radius enclosing half of the remnant mass. We also define line-of-sight stellar velocity dispersion of the remnants as in BoylanKolchin, Ma \& Quataert (2005). We use the surface mass densityweighted velocity dispersion within $R_{\mathrm{e}}$,

$\sigma_{\mathrm{e}}^{2}=\frac{\int_{2 \epsilon}^{R_{\mathrm{e}}} \sigma_{\mathrm{los}}^{2}(R) \Sigma(R) R \mathrm{~d} R}{\int_{2 \epsilon}^{R_{\mathrm{e}}} \Sigma(R) R \mathrm{~d} R}$,

where $\Sigma(R)$ is the surface mass density and $\sigma_{\text {los }}$ is the line-of-sight velocity dispersion. We use $2 \epsilon$ as the lower limit of the integrations in equation (8), where $\epsilon$ is the softening length. Taking into account projection effects, we average $R_{\mathrm{e}}$ and $\sigma_{\mathrm{e}}$ over 100 random projections.

To derive the major and minor axes of merger remnants, we obtain the principal axes of the merger remnants using the reduced moment-of-inertia tensor (Dubinski \& Carlberg 1991; BoylanKolchin et al. 2005):

$\begin{aligned} I_{i j} & =\sum_{l=1}^{N} \frac{x_{l, i} x_{l, j}}{a_{l}^{2}}, \\ a_{l} & =\left(x^{2}+\frac{y^{2}}{q^{2}}+\frac{z^{2}}{s^{2}}\right)^{1 / 2},\end{aligned}$ 
where $a_{l}$ is the elliptical radius and $q$ and $s$ are the axis ratios with $s \leqq q \leqq 1 . q$ and $s$ are calculated through

$q=\left(\frac{I_{y y}}{I_{x x}}\right)^{1 / 2}$ and $s=\left(\frac{I_{z z}}{I_{x x}}\right)^{1 / 2}$,

where $I_{x x}, I_{y y}$ and $I_{z z}$ are the principal components of the tensor. $N$ is the number of particles in the remnants, defined as all stellar particles within $r_{\text {trunc }}$. The procedure in determining these principle axes has to be performed iteratively, starting with the assumption of spherical symmetry, $q=s=1$.

\section{RESULTS}

We have run $N$-body simulations of the dry major, simultaneous minor and sequential minor mergers as described in Section 3. Physical quantities of the merger remnants are summarized in Table 2.

\subsection{Snapshots}

Fig. 5 shows five snapshots for three runs: 1A10Bsm, 1A10Bsq and 1A10Csq (Table 2). The elapsed time from the beginning of each run is written in the box of each snapshot. As described in Section 3 , we stop runs after $t=3.6 \mathrm{Gyr}$ of evolution in the minor merger simulations and analyse the merger remnants at $t=3.5$ 3.6 Gyr, to allow a small number of surviving satellites to be among the remnants.

In this final state, there are several surviving cores of satellite stellar systems among the remnants of the 10 minor mergers. However, most stars and the dark matter components of the satellite galaxies have already been stripped or merged into the primary galaxies. We show that these surviving cores have only a small effect on the properties of the merger remnants in Section 4.3. In the major merger (run 2A), the first crossing of galaxies is at $\sim 0.5 \mathrm{Gyr}$ and the merger appears to be completed by $2.3 \mathrm{Gyr}$.

\subsection{Density profile}

One of the most revealing properties of the remnants is their density profiles. Fig. 6 shows the angle-averaged stellar density profiles of the merger remnants for five runs. In this figure, we show three types of final density profiles for each run: the profiles of the stars that initially belong to the primary galaxy (we call these 'primary stars'), the stars that initially belong to the satellite galaxies (we call these 'satellite stars') and all the stars. For comparison, the density profile of the initial galaxy model is also shown. The density profiles for all the stars differs significantly between each run. Inside the inner regions of the half-mass radii (summarized in Table 2), the gravity of the stellar mass is closely related to the velocity dispersion of the stars in this region, as shown in Section 4.4. Below, we discuss these differences in each of the inner and outer sections of the remnants.

\subsubsection{Inner region of the stellar system}

To make clear the difference between the five runs in the inner regions, we show the cumulative mass distributions of the star particles of the remnants in Fig. 7. We first focus on the distributions of the primary stars. Comparing the distribution of primary stars of the initial state with that of the final state for minor mergers of compact satellites (runs 1A10Bsm, 1A10Bsq, and 1A10Dsq), we show that the decrease in the density profiles is due to expansion of the primary stars. Such an expansion is caused by dynamical friction heating from the satellites (El-Zant, Shlosman \& Hoffman 2001; Ma \& Boylan-Kolchin 2004). We see in Fig. 7 that this expansion is smaller for those minor mergers with diffuse satellites (runs 1A10Csm and 1A10Csq). As shown in Fig. 5, compact satellites can survive for a longer time-scale than diffuse satellites in the tidal field of the primary galaxy (see also Boylan-Kolchin \& Ma 2007). This increased lifetime means that dynamical friction heating can be more effective in expanding the primary stars. Therefore, compact satellites are more efficient at heating than diffuse satellites.

In the inner region, the density profiles of the satellite stars in the remnants depend on the compactness of the satellites. From Fig. 6 for compact satellites, we can see that there are more satellite stars within the half-mass radii than for diffuse satellites. Once again, this is due to compact satellites surviving against the tidal field of the primary galaxy to reach its inner regions. For those minor mergers with diffuse satellites, the satellite stars have a smaller contribution to the density profiles within the half-mass radii. This is because diffuse satellites are easily disrupted by tidal stripping and tidal shocking from the primary galaxy. This result is consistent with the minor merger simulations of a single satellite galaxy by Boylan-Kolchin \& Ma (2007).

Compared with sequential minor mergers, simultaneous minor mergers find a higher number of satellite stars within the halfmass radii. It is known that in a time-varying self-gravitational potential particles exchange their energies with each other, altering the energy distribution of the particles. This process is known as violent relaxation (Lynden-Bell 1967) and produces tightly bound particles. Section 4.4 will show in more detail that the satellite stars of simultaneous minor mergers have experienced this process. We will also show in Section 4.4 that in the case of sequential minor mergers the violent relaxation process is less effective and the number of tightly bound particles is smaller than for simultaneous minor mergers.

\subsubsection{Outer region of the stellar system}

Fig. 6 shows that the increase in density in the outer regions of the density profile is dominated by the accretion of satellite stars on to the outside of the half-mass radii. The density profiles of the primary stars is hardly changed, as shown in Fig. 6, and follows a $r^{-4}$ profile in the outer region. On the other hand, the slope of the density profiles of the satellite stars follows an $r^{-3}$ profile up to several $100 \mathrm{kpc}$. Then, for radii larger than several $100 \mathrm{kpc}$, the slope declines as $\rho \propto r^{-4}$. These differences show that the two components have distinct origins: an already formed system and a newly accreted system. In particular, in the case of the sequential minor mergers of diffuse satellites (run 1A10Csq), the satellite stars contribute to the envelope of the density profile of the remnant as an excess component, as shown in Fig. 6. Similar results are obtained by Abadi, Navarro \& Steinmetz (2006). Abadi et al. (2006) simulate an isolated disc galaxy with cosmological initial conditions and show that the density profile of the accreted stellar component follows the slope $\rho \propto r^{-3}$ near the edge of the primary stellar component of the galaxy. In addition, the slope reaches $\rho \propto r^{-4}$ or steeper near the virial radius of the system. Several recent observations of compact massive ETGs at low $z(z<0.5)$ show that the surfacebrightness profiles of the ETGs are well fitted with two-component Sérsic profiles (Stockton, Shih \& Larson 2010; Shih \& Stockton 2011). While these Sérsic indexes are relatively small $(n \lesssim 2)$, these galaxies may have experienced size growth by minor mergers like our simulations. 

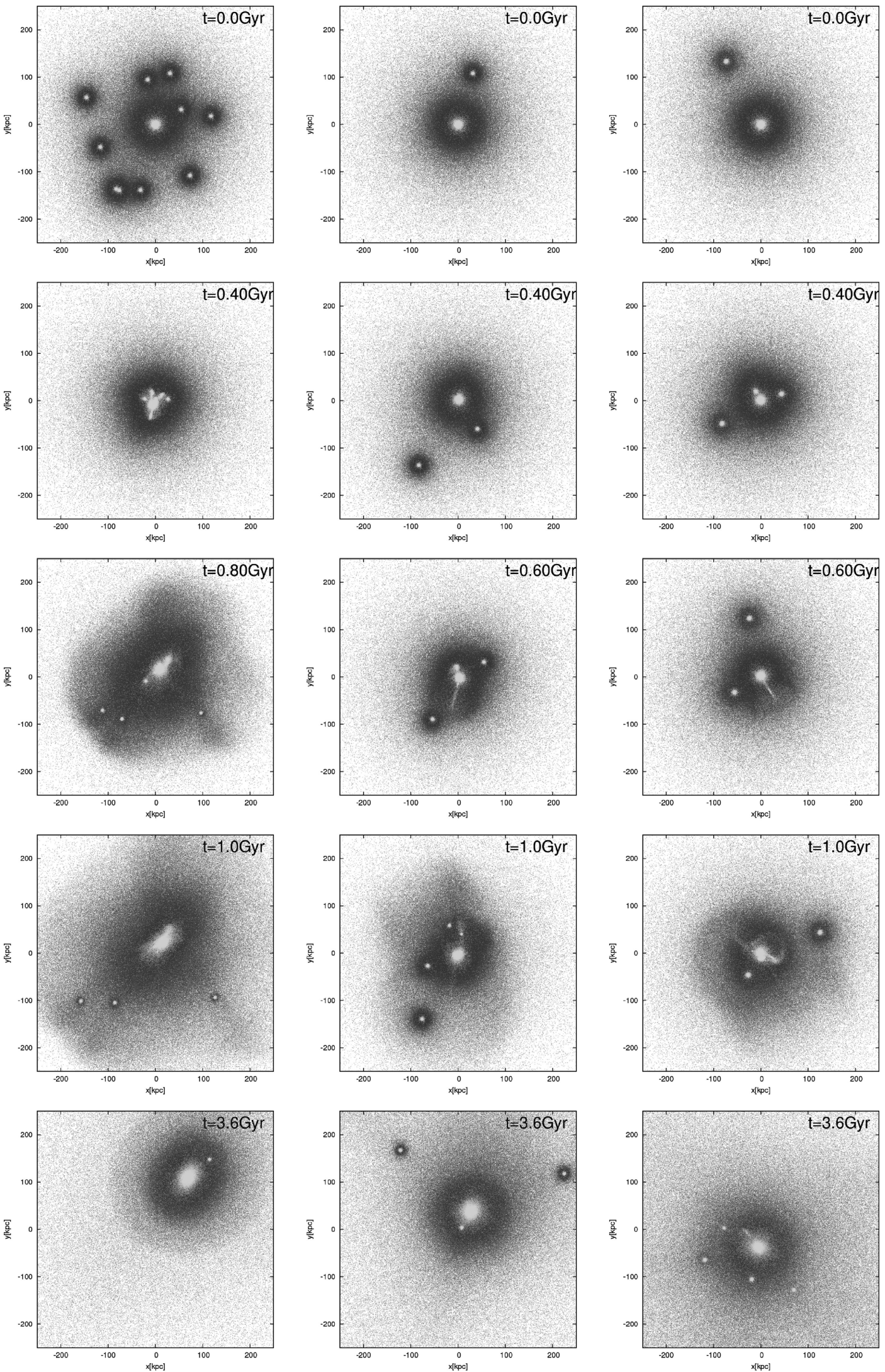

Figure 5. Snapshots of the particles of the three runs on the $x-y$ plane. Left-hand panels: simultaneous minor mergers of compact satellites, run 1A10Bsm. Middle panels: sequential minor mergers of compact satellites, run 1A10Bsq. Right-hand panels: sequential minor mergers of diffuse satellites, run 1A10Csq. 

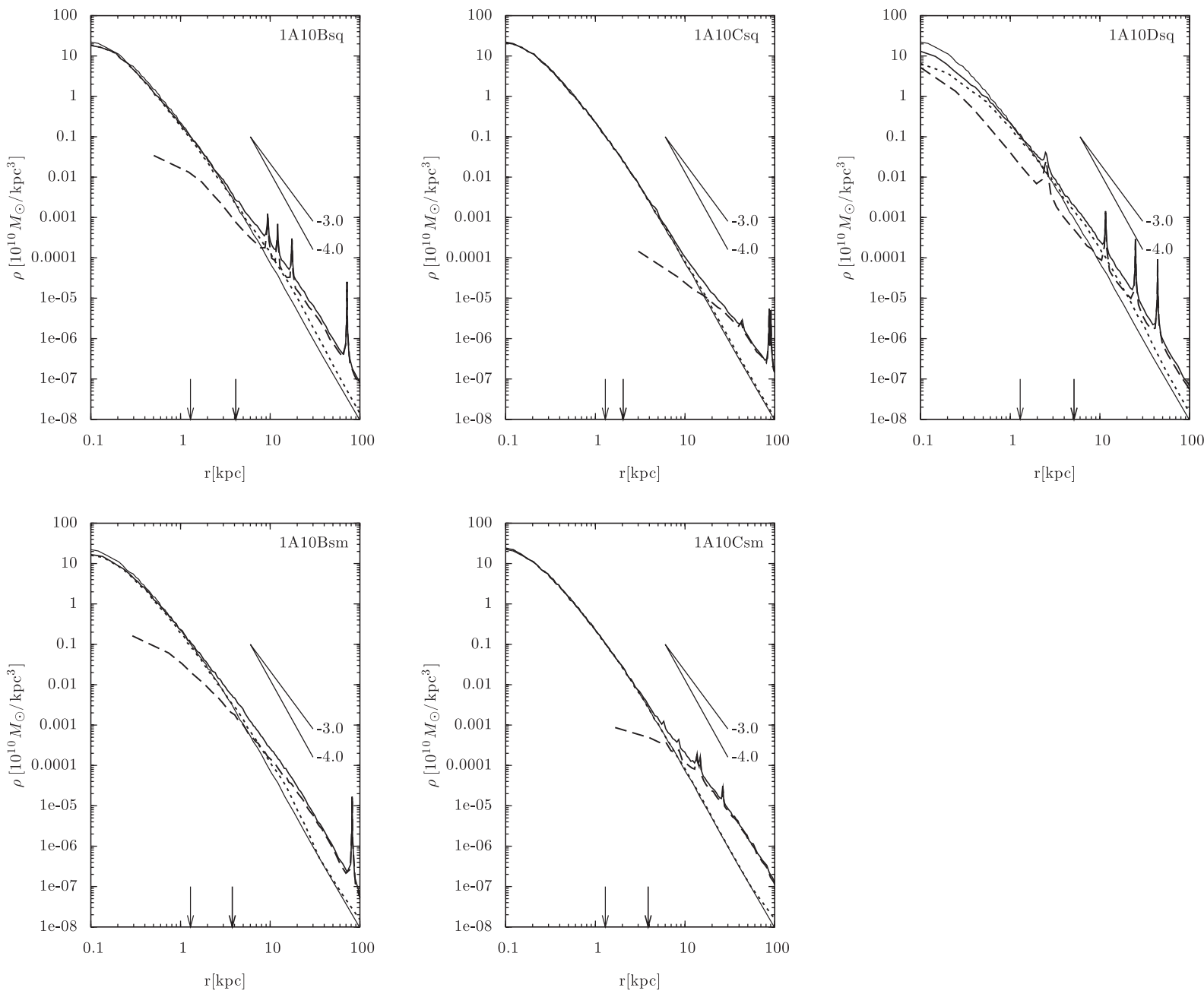

Figure 6. The angle-averaged density profiles of the remnant stellar systems of runs. From top left to bottom right: 1A10Bsq, 1A10Csq, 1A10Dsq, 1A10Bsm and $1 \mathrm{~A} 10 \mathrm{Csm}$. Thick solid lines are the density profiles of all stars of the merger remnants, dotted lines denote stars that belonged to the primary galaxy (primary stars), and dashed lines denote stars that belonged to the satellite galaxies (satellite stars). Thin lines show the initial galaxy model. Slopes of $r^{-3.0}$ and $r^{-4.0}$ are also shown for comparison. Thick arrows are half-mass radii of the stellar systems of the remnants. Thin arrows are half-mass radii of the stellar system of the initial primary galaxy model.

\subsubsection{Dark matter halo}

Fig. 7 also shows cumulative mass distributions of dark matter particles of the merger remnants of the five runs. The masses of dark matter haloes are smaller than the stellar mass in the inner regions. However, the change in dark matter mass distribution is important, since this affects the velocity dispersion of the stars in this region, as shown in Section 4.4. In the case of minor mergers of compact satellites, the dark matter particles that initially belong to the primary galaxy (we call these 'primary DMs') expand due to dynamical friction heating. Compared with simultaneous minor mergers, sequential minor mergers have only a small number of dark matter particles that initially belong to satellite galaxies (we call these 'satellite DMs') and contribute to the density profiles within $10 \mathrm{kpc}$ from the centre of the merger remnant. For simultaneous minor mergers, a significant amount of the satellite DM is deposited in the central $10 \mathrm{kpc}$. Thus, sequential minor mergers of compact satellites are the most efficient in expanding the dark matter distribution and in decreasing the central dark matter density.

\subsection{Stellar surface-density profile}

We show the angle-averaged stellar surface-density profiles of the merger remnants in Fig. 8. For the evolution in surface-density profiles, the choice of projection direction does not significantly alter the result. For the major merger (run 2A), the surface densities within $r \lesssim 1 \mathrm{kpc}$ increase from the initial state. On the other hand, for the minor mergers the surface densities are approximately stationary within $r \lesssim 1 \mathrm{kpc}$. For $\gtrsim 2 \mathrm{kpc}$, the growth in surface densities is dominated by the accretion of the satellites on to the outer envelope. The latter change agrees well with the recent observational results of van Dokkum et al. (2010) and is an example of 'inside-out growth'. These profiles depend on the compactness of the satellite galaxies. In Fig. 8, we show that inside-out growth is significant when the satellite galaxies are diffuse.

In the case of the 10 sequential minor mergers, there are several surviving cores of satellite stellar systems within $r_{\text {trunc }}$ of the remnants. Due to these cores, the remnant sizes might be artificially large in our analysis. We examine the effects of these cores 

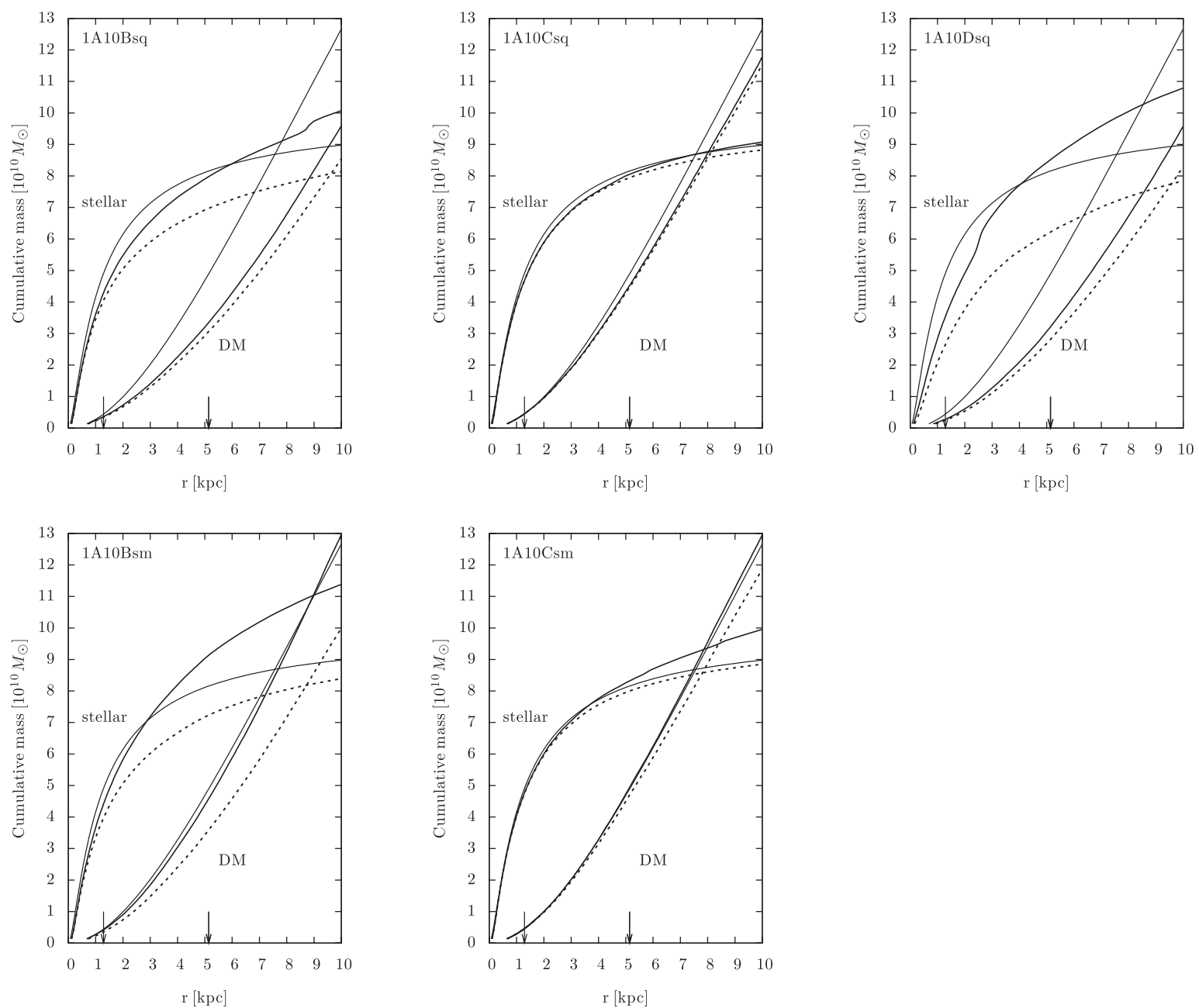

Figure 7. Cumulative mass distribution of the remnant stellar systems and dark matter particles. From top left to bottom right: 1A10Bsq, 1A10Csq, 1A10Dsq, $1 \mathrm{~A} 10 \mathrm{Bsm}$ and $1 \mathrm{~A} 10 \mathrm{Csm}$. Thick solid lines are the cumulative mass distributions of all stars or all dark matter particles of the merger remnants, dotted lines denote primary stars or primary dark particles. Thin lines denote the initial galaxy model. Thick arrows are half-mass radii of the stellar systems of the remnants. Thin arrows are half-mass radii of the stellar system of the initial primary galaxy model.
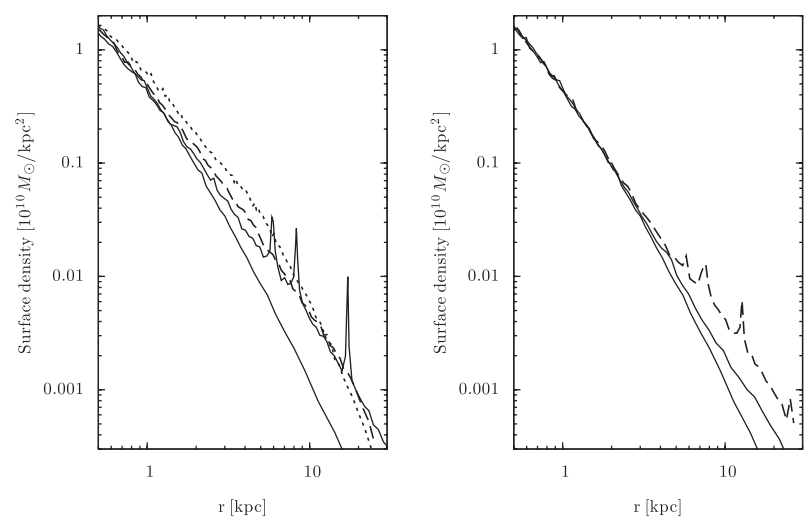

Figure 8. Left: angle-averaged surface-density profiles of the remnant stellar systems in one projection, 2A (dotted), 1A10Bsm (dashed), 1A10Bsq (thick solid) and initial model A (thin solid). Right: the same as the left panel, but for 1A10Csm (dashed), 1A10Csq (thick solid) and initial model A (thin solid). in determining the sizes and mass of merger remnants by removing the satellite cores from the surface-density profile of run $1 \mathrm{~A} 10 \mathrm{Bsq}$. We find that the remnant after the removal is $\sim 0.67 \times 10^{10} \mathrm{M}_{\odot}$ less massive and $\sim 0.32 \mathrm{kpc}$ smaller than with the cores included. This size change is much smaller than the size growth of the remnant of 1A10Bsq, which increased from 1-3 kpc. Therefore, in our analysis, these cores do not affect the size growth of the remnants significantly.

\subsubsection{Sérsic-profile fitting}

In order to compare the structures of our merger remnants quantitatively with observational results, we fit the stellar surface-density profiles of the remnants along the major and the minor axes derived by the method in Section 3.5 with the Sersic (1968) $R^{1 / m}$ law:

$I(R)=I_{0} \exp \left[-b(m)\left(\frac{R}{R_{\mathrm{e}}}\right)^{1 / m}\right]$,

where $b(m) \sim 2 m-1 / 3+4 /(405 m)$ (Ciotti \& Bertin 1999). We choose the radial range $[0.1,10] \mathrm{kpc}$ for this fitting. In this range, the 
Table 4. The Sérsic index. The $b-c$ plane means the projection along the major axis. The remnant is projected in the plane with intermediate axis $b$ and minor axis $c$. The $a-b$ plane is the projection along the minor axis. This plane contains the major axis $a$ and the intermediate axis $b$.

\begin{tabular}{ccc}
\hline Name & $b-c$ plane & $a-b$ plane \\
\hline A (initial) & 4.07 & 4.04 \\
2A & 5.00 & 6.16 \\
1A10Bsm & 6.51 & 6.32 \\
1A10Bsq & 11.3 & 8.47 \\
1A10Csm & 8.41 & 8.27 \\
1A10Csq & 4.83 & 4.76 \\
1A10Dsq & 4.66 & 7.87 \\
\hline
\end{tabular}

surface-density profiles are well fitted with a single Sérsic profile. We note that the Sérsic index $m$ is very sensitive to the fitting range (see also Boylan-Kolchin et al. 2005).

We find that the Sérsic indexes of the remnants increase from our initial model in all runs (Table 4). These values are consistent with observed massive ETGs (e.g. Bertin, Ciotti \& Del Principe 2002; Graham \& Guzmán 2003). In particular, for run 1A10Bsq, the Sérsic index increases significantly because of the marked insideout growth. On the other hand, for run 1A10Csq the Sérsic index increases less. As described above, this is because the stars of diffuse satellites are easily stripped by the tidal fields of the primary galaxies, and few satellite stars are added inside $10 \mathrm{kpc}$ from the centre of the remnant. The increase of Sérsic index depends significantly on the compactness of the satellites and the manner of minor merger (sequential or simultaneous).

\subsection{Velocity-dispersion profile}

In Fig. 9 we show the evolution of the line-of-sight stellar velocitydispersion profiles for the four runs with minor mergers. As with the surface-density profiles, the choice of projection does not affect our main results. Fig. 9 shows that in the case of minor mergers with compact satellites, the velocity dispersion of the stellar systems in the central region decreases from the initial model more strongly than for minor mergers with diffuse satellites. This is because the central region is gravitationally heated by the compact satellites. We compare the velocity dispersion of the remnant of run $1 \mathrm{~A} 10 \mathrm{Bsm}$ with that of run $1 \mathrm{~A} 10 \mathrm{Bsq}$ at $\sim 2.4 \mathrm{kpc}$, which is near the effective radii of these remnants. It is obvious from Fig. 9 that for run 1A10Bsq the velocity dispersion within this radius is smaller than for run $1 \mathrm{~A} 10 \mathrm{Bsm}$. This is because for run 1A10Bsq few satellite stars and satellite DMs are deposited within this radius. For run 1A10Bsm, relatively large amounts of satellite stars and satellite DMs are deposited within this radius, and the velocity dispersion of the remnant increases from the initial value in this region. Therefore, in the case of sequential minor mergers of compact satellites, the velocity dispersion is smaller in this region.

To interpret the difference in the mass deposited during both runs, we show the differential energy distributions $N(E)$ for the initial and final stellar components in Fig. 10, where $N(E) \mathrm{d} E$ represents the number of particles of specific energy between $E$ and $E+\mathrm{d} E$. For the simultaneous minor merger case, the distribution of satellite stars broadens during the mergers and low-energy stars are more bound than the initial state. This broadening of $N(E)$ is similar to the behaviour of $N(E)$ in cold-collapse simulations (Binney \& Tremaine 2008). This means that in this case violent relaxation is effective for the satellite stars. Due to such evolution, the satellite stars are more bound and invade the centre of the primary galaxy strongly. On the other hand, there is smaller change of $N(E)$ for the sequential minor merger case. This means that in this case violent relaxation is not effective and the satellite stars cannot invade the inner region. In both simulations shown in Fig. 10, the number of those primary stars that are most bound is reduced. This unbinding is induced by dynamical friction heating during minor mergers.
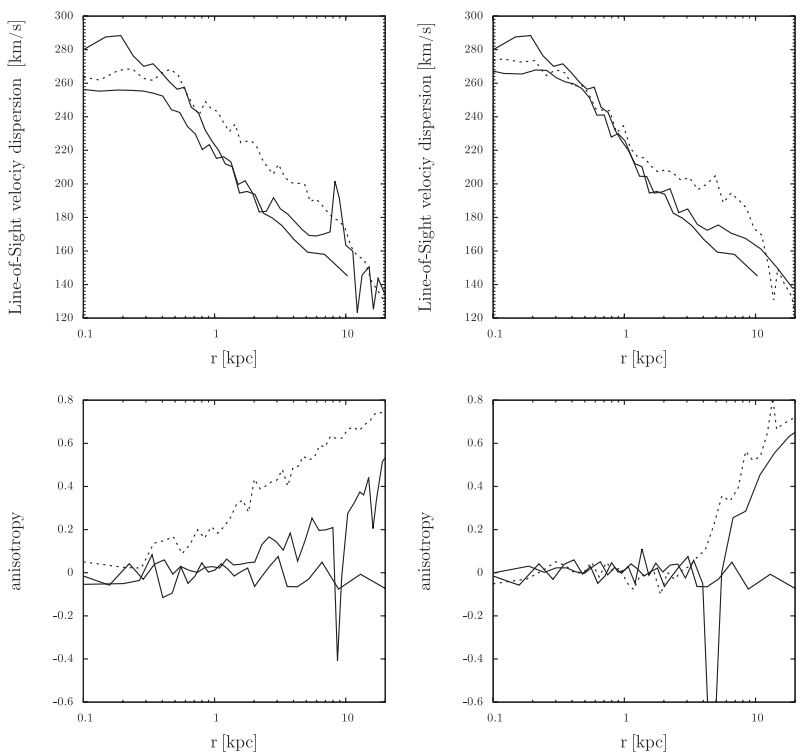

Figure 9. Top left: line-of-sight velocity-dispersion profiles of the remnant stellar systems, 1A10Bsm (dotted), 1A10Bsq (thick solid) and initial model A (thin solid). Top right: the same as the top-left panel, but for 1A10Csm (dotted), 1A10Csq (thick solid) and initial model A (thin solid). Bottom: anisotropy parameter $\beta$ for the merger remnants in the top panels.
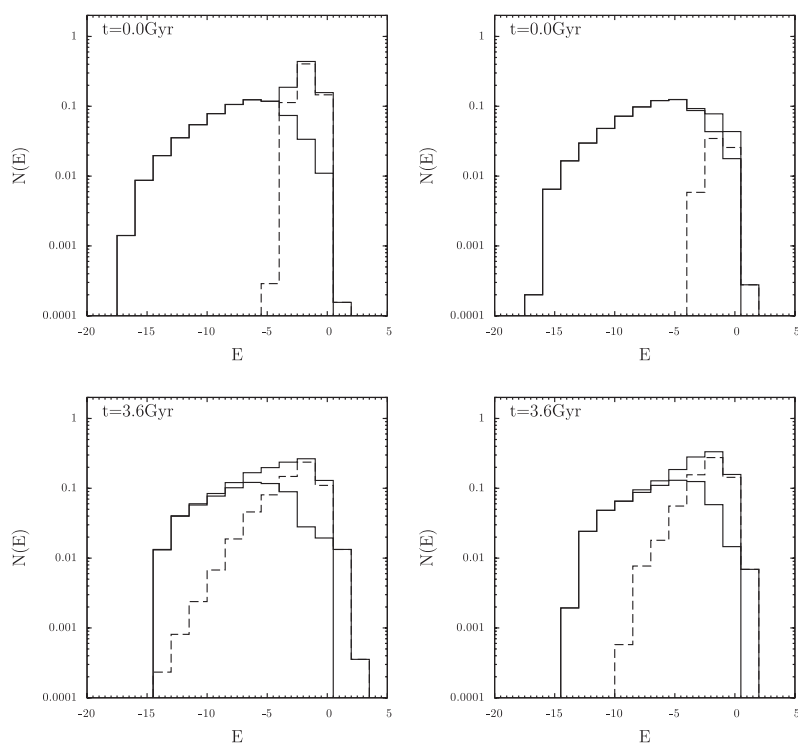

Figure 10. Left-hand panels: initial (top panel) and final (bottom panel) differential energy distributions of star particles for the simultaneous minor mergers of compact satellites (run 1A10Bsm). The thick solid, thin solid and dashed lines refer to the distributions of total stars, primary stars and satellite stars, respectively. The energy per unit mass $E$ is normalized to the internal unit of our simulations. Right hand panels: same as left-hand panels, but for the sequential minor mergers of compact satellites (run 1A10Bsq). 
We also investigate the anisotropy of the velocity dispersions of stellar merger remnants. We measure an anisotropy parameter for the remnants

$\beta=1-\left(\frac{\sigma_{\theta}^{2}}{\sigma_{r}^{2}}\right)$,

where $\sigma_{\theta}$ and $\sigma_{r}$ are the azimuthal and radial velocity dispersions with respect to the centre of the remnants, respectively. Here, we assume $\sigma_{\theta}=\sigma_{\phi}$. In the bottom panels of Fig. 9, we show the anisotropy of the merger remnants. For all runs, radial anisotropy increases with radius. It is obvious that in the case of sequential minor mergers, radial anisotropy is smaller than for simultaneous minor mergers. This result is consistent with that of Athanassoula (2005), who shows that radial anisotropy becomes smaller for larger time intervals between mergers in sequential galaxy mergers in galaxy groups. We note that, for sequential minor mergers, satellite orbits are radial with respect to the 'initial' position of the primary galaxy and in actuality these orbits may be more circular than for simultaneous minor mergers. This may affect the small radial anisotropy for sequential minor mergers. Our results suggest that the manner of minor merger influences the radial anisotropy of merger remnants.

\subsection{Evolution of stellar mass, $R_{\mathrm{e}}$ and $\sigma_{\mathrm{e}}$}

We summarize the results for stellar masses, effective radii $R_{\mathrm{e}}$ and velocity dispersions $\sigma_{\mathrm{e}}$ of the merger remnants in Table 2. The minor mergers of compact satellites are more efficient in increasing the mass of the remnants than those of diffuse satellites. More than half of the satellite stars in compact satellites contribute to the mass of the remnants. On the other hand, in the case of diffuse satellites (model C) the satellites are disrupted by tidal stripping and shocking during the merging and most satellite stars of diffuse satellites are distributed in the envelope of the remnants. These are too diffuse to observe and do not contribute to the mass of the remnants (run 1A10Csq).

Changes of $R_{\mathrm{e}}$ and $\sigma_{\mathrm{e}}$ are influenced by both the manner of the minor mergers and the compactness of the satellite galaxies. For minor mergers of compact satellites, sequential cases cause larger size growth and a greater decrease in the velocity dispersion compared with simultaneous cases. On the other hand, for minor mergers with diffuse satellites, simultaneous cases cause larger size growth, while the decrease in velocity dispersion is weak in both sequential and simultaneous cases. There are two processes that are closely related to the changes of $R_{\mathrm{e}}$ and $\sigma_{\mathrm{e}}$. The first is the expansion of the primary stars by dynamical friction heating. This increases the size of the primary galaxy and decreases the velocity dispersion, if it is principally determined by the mass distribution of the primary stars. In the case of minor mergers with compact satellites, this expansion is more effective in both sequential and simultaneous cases than for minor mergers with diffuse satellites (Fig. 7). The second process is the mass deposit of satellite stars by minor mergers. The mass deposit increases the size of the remnant efficiently for all runs. It is important for changes to the velocity dispersion whether the satellite stars are deposited within the effective radii of the merger remnants or beyond them. If a significant number of satellite stars are deposited within the effective radii, the velocity dispersions of the remnants increase. In the case of sequential minor mergers, mass deposit within the effective radii is small. In this case, the velocity dispersion is mainly determined by the expansion of the primary stars and hence the velocity dispersion decreases. These two processes also cause changes in the density and velocity-dispersion profiles (see Sections 4.2 and 4.4). We note that comparison of runs
1A10Bsq, 1A10Csq and 1A10Dsq shows that the effective radii increase more for minor mergers of compact satellites than for minor mergers of diffuse satellites. This result is apparently contrary to expectations based on the virial theorem and energy conservation (e.g. Naab et al. 2009). However, because of tidal disruption of diffuse satellites as described above, the size and mass increase are weak in the case of diffuse satellites.

We present two important relations from our results. The first is the relation between size growth and mass growth. The second is the relation between decrease in velocity dispersion and mass growth. We parametrize the relation between the size growth and the mass growth as

$$
\frac{R_{\mathrm{e}, \mathrm{f}}}{R_{\mathrm{e}, \mathrm{i}}}=\left(\frac{M_{\mathrm{f}}}{M_{\mathrm{i}}}\right)^{\alpha}
$$

to quantify the efficiency of the size growth (hereafter we call $\alpha$ the size growth efficiency), where $R_{\mathrm{e}, \mathrm{i}}$ and $R_{\mathrm{e}, \mathrm{f}}$ are the initial and final effective radius, while $M_{\mathrm{i}}$ and $M_{\mathrm{f}}$ are the initial and remnant stellar mass, respectively. We also parametrize the relation between the change of the velocity dispersion and the stellar mass growth as

$\frac{\sigma_{\mathrm{e}, \mathrm{f}}}{\sigma_{\mathrm{e}, \mathrm{i}}}=\left(\frac{M_{\mathrm{f}}}{M_{\mathrm{i}}}\right)^{-\gamma}$

to quantify the efficiency in the change in velocity dispersion. Table 2 shows $\alpha$ and $\gamma$ for each run. As shown in Table 2, for the cases of sequential minor mergers $\alpha$ and $\gamma$ are generally higher than that for simultaneous minor mergers. In particular, we show that the sequential minor mergers of model $\mathrm{B}$ are the most efficient in size growth, $\alpha \simeq 2.7 . \gamma$ is larger for runs with model $\mathrm{D}$, the most compact satellite. In the case of sequential minor mergers of diffuse satellites (run 1A10Csq), $\alpha$ and $\gamma$ are also larger. In this case, only the expansion is effective. However, here the satellite stars do not contribute to the mass increase of the merger remnant because of tidal disruption of the diffuse satellites as described above. Therefore, minor mergers of diffuse satellites do not contribute to size growth significantly.

As described in the previous section, in the case of 10 sequential minor mergers there are several surviving cores from satellite stellar systems in the remnants within $r_{\text {trunc }}$. Using the prescription for removing satellite cores for $1 \mathrm{~A} 10 \mathrm{Bsq}$, we find that size growth efficiency $\alpha \simeq 2.7$ with or without a satellite core. Therefore in our analysis, these cores do not affect the size growth efficiency of the remnants significantly.

\section{DISCUSSION}

\subsection{Size growth efficiency}

As shown in Table 2, the size growth efficiencies $\alpha$ are higher than for an analytical estimation (e.g. Naab et al. 2009) or than found numerically in previous works (e.g. Boylan-Kolchin et al. 2006). We check these high efficiencies using a simple analytical argument: Under the assumptions of energy conservation, single-component galaxies and a parabolic orbit in a minor merger, Naab et al. (2009) show that the ratio of the final to initial gravitational radius is

$\frac{r_{\mathrm{g}, \mathrm{f}}}{r_{\mathrm{g}, \mathrm{i}}}=\frac{(1+\eta)^{2}}{(1+\eta \epsilon)}$.

Here, we define $\eta \equiv M_{2} / M_{1}$ and $\epsilon \equiv\left\langle v_{1}^{2}\right\rangle /\left\langle v_{2}^{2}\right\rangle$, where $M_{1}$ and $\left\langle v_{1}^{2}\right\rangle$ are the mass and velocity dispersion of the more massive galaxy before the minor merger and $M_{2}$ and $\left\langle v_{2}^{2}\right\rangle$ are those of the less massive galaxy. This formula is also valid for two-component galaxy 
Table 5. Physical properties of progenitors and merger remnants in binary minor merger simulations. Note that we define the merger remnants as bound particles here. Each column is similar to Table 2 except for the fifth column, which gives the structure parameter of the galaxy.

\begin{tabular}{lcccc}
\hline Name & $\begin{array}{c}\text { Stellar mass } \\
\left(\mathrm{M}_{\odot}\right)\end{array}$ & $\begin{array}{c}R_{\mathrm{e}} \\
(\mathrm{kpc})\end{array}$ & $\alpha$ & $f$ \\
\hline modelA & $9.97 \times 10^{10}$ & 0.995 & & 0.173 \\
modelB & $0.996 \times 10^{10}$ & 0.453 & & 0.177 \\
modelC & $0.994 \times 10^{10}$ & 1.11 & & 0.173 \\
modelD & $0.999 \times 10^{10}$ & 0.261 & & 0.163 \\
1A1B & $10.8 \times 10^{10}$ & 1.21 & 2.40 & 0.185 \\
1A1C & $10.7 \times 10^{10}$ & 1.16 & 2.20 & 0.183 \\
1A1D & $10.9 \times 10^{10}$ & 1.23 & 2.54 & 0.183 \\
\hline
\end{tabular}

models if the dark matter halo profile strictly follows the stellar density profile (Nipoti et al. 2012). We adopt this assumption in the following discussion. $r_{\mathrm{g}}$ in equation (16) can be replaced with $R_{\mathrm{e}}$, where $r_{\mathrm{g}}$ and $R_{\mathrm{e}}$ satisfy the following equation:

$E \equiv-f \frac{G M_{*}^{2}}{R_{\mathrm{e}}}=-\frac{1}{2} \frac{G M_{*}^{2}}{r_{\mathrm{g}}}$,

where $E$ is the energy of the stellar system and $f$ is a structural parameter that depends on the stellar and dark matter density profiles. We obtain

$\frac{R_{\mathrm{e}, \mathrm{f}}}{R_{\mathrm{e}, \mathrm{i}}}=\frac{f_{\mathrm{f}}}{f_{1}} \frac{(1+\eta)^{2}}{(1+\eta \epsilon)}$,

where $f_{1}$ and $f_{\mathrm{f}}$ are the structural parameters of the progenitor and remnant stellar systems. Here, we assume that the two progenitors have the same structural parameter $f_{1}$. This assumption is valid for runs $1 \mathrm{~A} 1 \mathrm{~B}$ and $1 \mathrm{~A} 1 \mathrm{C}$. Thus, if $f$ increases in a dry minor merger, $R_{\mathrm{e}}$ increases more than for the case where $f_{\mathrm{f}}=f_{1}$. From equation (18), the size growth efficiency can be written as

$\alpha=\frac{\mathrm{d} \ln R_{\mathrm{e}}}{\mathrm{d} \ln M_{*}}=\frac{\ln \left(f_{\mathrm{f}} / f_{1}\right)}{\ln (1+\eta)}+2-\frac{\ln (1+\eta \epsilon)}{(1+\eta)}$.

We estimate $\alpha$ from $f_{\mathrm{f}} / f_{1}, \eta$ and $\epsilon$ from our numerical results with equation (19). In the case of run $1 \mathrm{~A} 1 \mathrm{~B}, \alpha=2.2$, since $f_{\mathrm{f}} / f_{1}=$ $1.07, \eta=0.1$ and $\epsilon=0.446$. For run 1A1C, $\alpha=2.3$, since $f_{\mathrm{f}} / f_{1}=$ $1.06, \eta=0.1$ and $\epsilon=0.297$. For run $1 \mathrm{~A} 1 \mathrm{D}, \alpha=2.0$, since $f_{\mathrm{f}} / f_{1}=$ $1.06, \eta=0.1$ and $\epsilon=0.580$. We analyse the merger remnants of runs 1A1B, 1A1C and 1A1D (see Table 5), defining the merger remnants as bound particles that are consistent with the energy conservation argument here. We obtain size growth efficiencies of $\alpha=2.4$ (run $1 \mathrm{~A} 1 \mathrm{~B}$ ) and $\alpha=2.2$ (run 1A1C). These values agree well with the analytical argument. On the other hand, in the case of run 1A1D $\alpha=2.5$, which does not agree well with the analytical result. In this case, the change in the stellar and dark matter halo profiles is larger, due to strong dynamical friction heating. Thus, this disagreement may stem from the assumption in the analytical argument that the dark matter halo profile follows the stellar density profile, which fails to hold in this case. Alternatively, the disagreement may be because the assumption that the two progenitors have the same structural parameter $f_{1}$ is invalid in this case. Therefore, we suggest that our high size growth efficiency can be interpreted as an increase in the structural parameter $f$ of merger remnants (see also a similar analysis in Boylan-Kolchin et al. (2006)). Similar results of high size growth efficiency for minor mergers on radial orbits are shown in Nipoti et al. (2012).

\subsection{Comparison with observations}

Our main focus is whether dry minor mergers can explain the size evolution problem of ETGs and reproduce their local scaling relations, in particular the stellar mass-size and stellar mass-velocitydispersion relation. We compare our numerical results with these results here.

\subsubsection{Stellar mass-size relation}

In Fig. 11, we compare our numerical results with the local stellar mass-size relation given by the SDSS (Shen et al. 2003). We show that sequential minor mergers of compact satellites cause the most efficient size growth, while all results move compact massive ETGs toward the local relation in Fig. 11. Interestingly, sequential minor mergers have $\alpha \simeq 2.7$ (Table 2), which is a much larger value than that obtained via simple analysis based on the virial relation, $\alpha \sim$ 2 (Bezanson et al. 2009; Naab et al. 2009), with assumptions of energy conservation, single-component galaxies and parabolic orbits of minor mergers. Sequential minor mergers of compact satellites can evolve compact massive ETGs to the average size of ETGs in the local Universe by a factor of $\sim 2$ mass growth. As shown in Section 2, such mass growth is likely to occur for the majority of high- $z$ massive galaxies.

Our high growth efficiency is consistent with the observational constraint by Bezanson et al. (2009). They suggest that the size growth efficiency, $\alpha$, must be $\alpha \gtrsim 2$ in order to satisfy the constraint that the number density of descendants of high- $z$ compact massive ETGs does not exceed constraints imposed by the $z=0$ galaxy mass function. Our result for the size growth efficiency for sequential minor mergers, $\alpha \simeq 2.7$, is consistent with their results.

Newman et al. (2012) estimate the size evolution of ETGs using the Cosmic Assembly Near-infrared Deep Extragalactic Legacy Survey (CANDELS) and conclude that the observed rapid size growth from $z \simeq 2$ to $z \simeq 1$ cannot be explained by the dry minor merger scenario, if they assume $\alpha \sim 1.3-1.6$ as motivated by previous studies (e.g. Nipoti et al. 2009b). However, our results

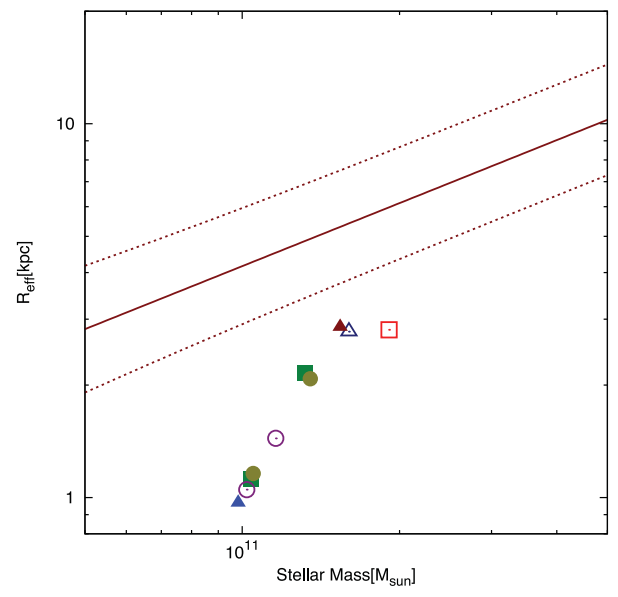

Figure 11. Stellar mass-size relation of merger remnants and the initial galaxy model. The solid triangle is for the initial galaxy model A. The empty square is for run $2 \mathrm{~A}$. The solid square is for run $1 \mathrm{~A} 10 \mathrm{Bsm}$. The empty circle is for run $1 \mathrm{~A} 10 \mathrm{Bsq}$. The filled circle is for run $1 \mathrm{~A} 10 \mathrm{Csm}$. The empty triangle is for run $1 \mathrm{~A} 10 \mathrm{Csq}$. The solid line shows the observed stellar masssize relation for ETGs (Shen et al. 2003), with the dispersion shown by the dotted lines. 
show a significantly higher size growth efficiency, $\alpha \simeq 2.7$, which may invalidate their conclusion.

\subsubsection{Stellar density}

As shown in Table 2, we find that the mean stellar densities within $1 \mathrm{kpc}, \bar{\rho}(<1 \mathrm{kpc})$, decrease by only a small factor in all runs. On the other hand, for sequential minor mergers of compact satellites, the mean stellar density within the effective radius, $\bar{\rho}\left(<R_{\mathrm{e}}\right)$, decreases by more than an order of magnitude. This evolution is consistent with several recent observational studies (Bezanson et al. 2009; van Dokkum et al. 2010), which show evidence to suggest that compact massive ETGs at high $z$ are the central stellar systems in normal nearby ETGs. This scenario is called the 'inside-out' growth of ETGs. We note that the decrease of $\bar{\rho}\left(<R_{\mathrm{e}}\right)$ in our results exceeds that from simple analysis ( $\mathrm{Naab}$ et al. 2009), corresponding to a size growth larger than that of the simple estimate.

\subsubsection{Stellar mass-velocity-dispersion relation}

In Fig. 12, we compare our results with the local stellar massvelocity-dispersion relation derived by Nipoti et al. (2009b). We find that the remnants of sequential minor mergers of compact satellites lie on the local relation and the velocity dispersions of all minor mergers decrease toward the local relation. We note that the decrease in velocity dispersion for almost all runs is less effective than that derived by simple analysis using the virial relation, $\gamma \sim$ $1 / 2$ (Naab et al. 2009).

\subsection{Size growth expected from the Millennium Simulation Data Base}

As shown in Section 2, we analyse the Millennium Simulation Data Base given by De Lucia \& Blaizot (2007) to derive the cumulative mass growth via minor mergers. In this subsection, we present the size growth for the sample galaxies derived by the Millennium Simulation Data Base, using the size growth formula given by equation (14) assuming the size growth efficiency $\alpha$. We confirm that for $\sim 95$ per cent $(\sim 91$ per cent) of our sample galaxies, $\sim 80$ per cent ( $\sim 90$ per cent) of the bulge mass at $z=0$ consists of stars that belonged to their progenitor galaxies. Thus, they mainly increase

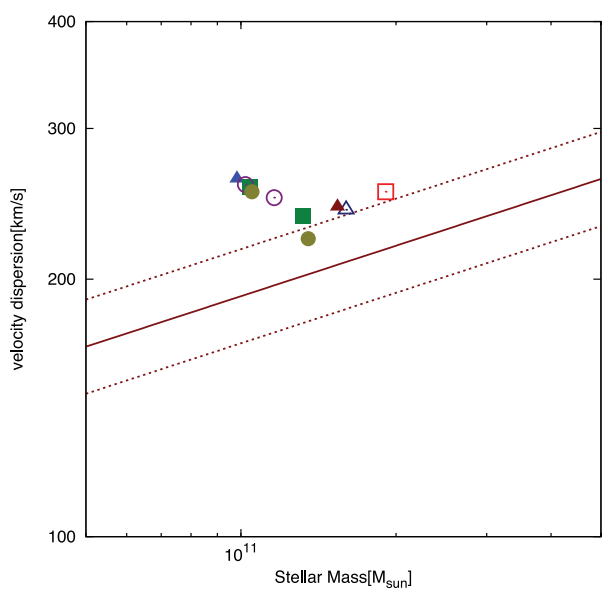

Figure 12. Stellar mass-velocity-dispersion relation of merger remnants and the initial galaxy model. Symbols are the same as in Fig. 11. The solid line shows the observed stellar mass-velocity-dispersion relation for ETGs (Nipoti et al. 2009b) with the $1 \sigma$ scatter shown by the dotted lines. their masses by dry mergers. This is mainly because De Lucia \& Blaizot (2007) use strong supernova and AGN feedback models. A small fraction of our sample galaxies increases their mass by star formation. These 'wet mergers' may influence their size increase. In this discussion, we neglect these 'wet mergers', since their number is small. Here, we divide the sample galaxies into two categories: brightest cluster galaxies (BCGs) and normal ETGs. We define the BCGs as those galaxies that are the central galaxies in FOF haloes with mass $\gtrsim 10^{14} \mathrm{M}_{\odot}$, otherwise we define galaxies as normal ETGs.

For the sample galaxies, we derive the average size growth factor $\left\langle R_{\mathrm{e}, \mathrm{f}} / R_{\mathrm{e}, \mathrm{i}}\right\rangle$ by the following procedure. First, for each sample galaxy we calculate the mass increase through minor mergers from $z=2.07$ to $z=0, \Delta m_{\text {minor }}$. Secondly, we calculate the mass growth factor $M_{\mathrm{f}} / M_{\mathrm{i}}$ by minor mergers from $z=2.07$ to $z=0$, where $M_{\mathrm{i}}$ is the bulge mass at $z=2.07$ and $M_{\mathrm{f}}=M_{\mathrm{i}}+\Delta m_{\text {minor }}$. Thirdly, we calculate the size growth factor $R_{\mathrm{e}, \mathrm{f}} / R_{\mathrm{e}, \mathrm{i}}$ with equation (14) for each sample galaxy, where we assume a size growth efficiency $\alpha$. Here, we adopt the same size growth efficiency for all sample galaxies. Finally, we average the size growth factors of all sample galaxies as $\left\langle R_{\mathrm{e}, \mathrm{f}} / R_{\mathrm{e}, \mathrm{i}}\right\rangle$.

We show the dependence of the average size growth factor on the adopted size growth efficiencies in Fig. 13. It is obvious that $\alpha \gtrsim 2.3$ is needed to explain the observed size evolution of ETGs from $z \sim 2$ to $z=0$ (a factor of three to five in size growth, e.g. Trujillo et al. 2006, 2007; Buitrago et al. 2008; Cimatti et al. 2008; van Dokkum et al. 2008). This constraint is consistent with the high size growth efficiency derived by our numerical simulations. On the other hand, the lower size growth efficiency $\alpha=1.3$ derived by the simultaneous dry minor merger simulations of Nipoti et al. (2009b) is insufficient to explain the observational results. Therefore, we suggest that in the dry minor merger scenario the sequential minor mergers we performed in this paper are responsible for the size evolution of ETGs.

We note that we use the maximum size growth efficiency in the above discussion, whereas the choice of $\alpha \simeq 2.7$ corresponds to the maximum predicted size increase and the assumptions that all minor mergers are those with mass ratios of $M_{2} / M_{1}<1 / 10$ and that their orbits are parabolic and head-on. In fact, there exists a wide range of mass ratios for minor mergers in cosmological merger histories. For example, Oser et al. (2012) find that the typical mass

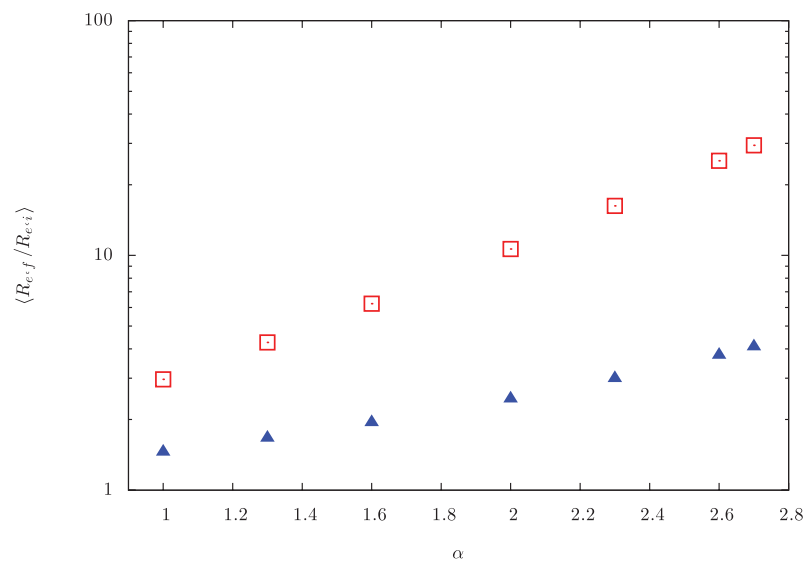

Figure 13. The average size growth factor $\left\langle R_{\mathrm{e}, \mathrm{f}} / R_{\mathrm{e}, \mathrm{i}}\right\rangle$ of our sample galaxies derived by the Millennium Simulation Data Base for various assumed size growth efficiencies. The derivation of $\left\langle R_{\mathrm{e}, \mathrm{f}} / R_{\mathrm{e}, \mathrm{i}}\right\rangle$ is described in the text. The horizontal axis shows the size growth efficiency $\alpha$, the empty square BCGs and the solid triangle normal ETGs. 
ratio of stellar mergers is 1:5 with cosmological hydrodynamical simulations. Additionally, there are satellite galaxies that have different properties, e.g. morphology, compactness and gas content, which may influence the size growth of compact massive ETGs. In future studies, we will take into account the mass ratio, orbits and many satellite properties for more robust predictions based on the dry minor merger scenario.

In Fig. 14, we show the individual size growth factor for all sample galaxies as a function of the bulge mass at $z=0$. Fig. 15 is the same as Fig. 14 but as a function of the FOF halo mass to which the each sample galaxy belongs at $z=0$. In this figure, we assume $\alpha=2.7$. We note that there are a considerable number of galaxies that have a small size growth factor from $z=2.07$ to $z=0$ from dry minor mergers (Fig. 14). These galaxies reside in dark matter haloes that have a wide range of mass, from galaxy-size haloes to cluster-size ones (Fig. 15), and almost all of them are normal ETGs. In addition, they do not increase their size by dry major mergers. In fact, of those galaxies that have a size growth factor less than $2, \sim 80$ per cent are minor-merger-dominated galaxies. Thus, these galaxies are expected to keep their size from $z \sim 2$. Such galaxies in cluster-size haloes may correspond to the nearby compact massive

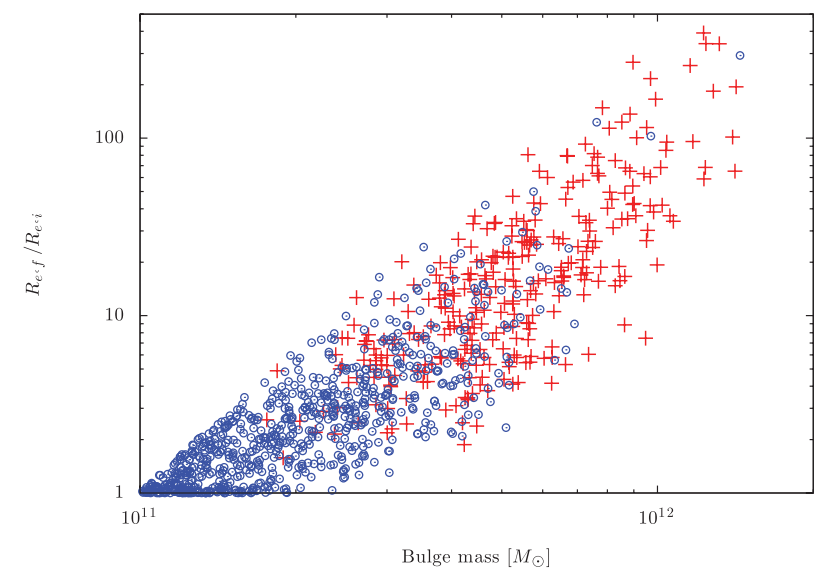

Figure 14. The size growth factor $R_{\mathrm{e}, \mathrm{f}} / R_{\mathrm{e}, \mathrm{i}} \equiv\left(M_{\mathrm{f}} / M_{\mathrm{i}}\right)^{\alpha}$ of our sample BCGs (crosses, red in the online article) and normal ETGs (open circles, blue in the online article) derived by the Millennium Simulation Data Base, where we assume $\alpha=2.7$, which is the most efficient case in our results. The horizontal axis shows the bulge mass at $z=0$. The vertical axis shows $R_{\mathrm{e}, \mathrm{f}} / R_{\mathrm{e}, \mathrm{i}}$.

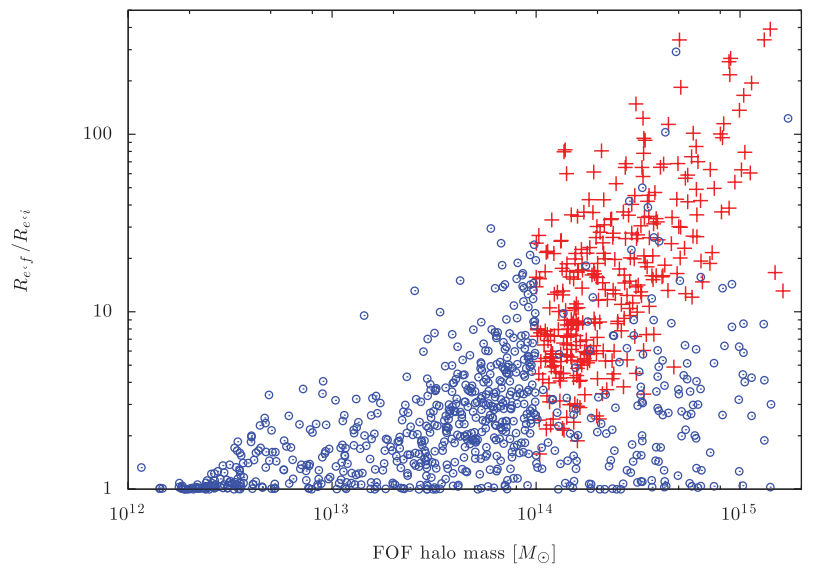

Figure 15. The same as Fig. 14, but for a horizontal axis showing the FOF halo mass to which the galaxies belong at $z=0$.
ETGs that Valentinuzzi et al. (2010) claim to find in nearby X-rayselected clusters.

\subsection{Comparison with previous work}

Our simulation results show a high size growth efficiency and a decrease in the velocity dispersion even in our lowest growth efficiency case with simultaneous minor mergers, compared with results from Nipoti et al. (2009b). This disagreement may be due to the initial conditions of the simulations. In this section, we examine the differences between our simulations and those performed by Nipoti et al. (2009b). Nipoti et al. (2009b) construct initial galaxy models that have a higher dark matter fraction within the stellar half-mass radius than our models. The correct dark matter fraction within the half-mass radii is uncertain for compact massive ETGs at high $z$. Sommer-Larsen \& Toft (2010) show that massive compact high- $z$ galaxies are strongly baryon-dominated in their inner regions through cosmological simulations. The semi-analytic model of Shankar et al. (2011) also predicts that the dark matter fraction of compact massive ETGs become smaller in at high $z$. Nipoti et al. (2009b) also assume a steeper inner slope, $\rho \propto r^{-1.5}$, than the Hernquist profile $\left(\rho \propto r^{-1}\right)$ for the stellar component. Their initial stellar model may preserve the inner structure during dry mergers and may bind satellite galaxies in the inner region. These lead to suppression of significant size growth and a lower decrease in the velocity dispersion. Nipoti et al. (2009b) assume more compact satellite galaxies than model B of our simulation. This is because they scale the satellite galaxies based on the fundamental plane derived by the SLACS survey. For model B in our simulations, we chose simply to scale down the primary galaxy (model A) with the same average density. This is because we assume that the formation epoch of model B is the same as that of model A, i.e. $z=2.0$, and that these models are expected to have the same average density. This assumption may mean that in dry minor merger simulations the effect of dynamical friction heating may be more effective than in our results.

\section{CONCLUSIONS}

We investigate the effects of sequential dry minor mergers on the size evolution of compact massive ETGs. From an analysis of the Millennium Simulation Data Base, we show that such minor mergers are highly likely in hierarchical structure formation. We perform $\mathrm{N}$-body simulations of sequential minor mergers with parabolic and head-on orbits, including dark matter and stellar components, and compare the properties of the merger remnants with those from simultaneous minor mergers. We show that sequential minor mergers are the most efficient for size growth and in decreasing the velocity dispersion of the stellar system. The evolution of stellar size and the velocity dispersion of the galaxy in sequential minor mergers with compact satellites agrees with recent observations. Furthermore, we construct the merger histories of candidates of high- $z$ compact massive ETGs using the Millennium Simulation Data Base and model the size growth of the galaxies via dry minor mergers. We can reproduce the mean size growth factor between $z=2$ and $z=0$ if we adopt the most efficient size growth efficiency $\alpha=2.7$ obtained in the case of sequential minor mergers in our simulations. Because these minor mergers are more common for galaxies in more massive FOF haloes (Fig. 2), our results suggest that, for ETGs in more massive dark matter haloes, size growth by dry minor mergers is still more effective. However, we note that our numerical result is valid for merger histories with typical mass ratios between $1 / 20$ 
and $1 / 10$ and with parabolic and head-on orbits. In addition, our most efficient size growth efficiency is likely an upper limit, since it is based on simulations in which the satellites have smaller mass ratios and are on radial orbits. In future studies, we will investigate various mass ratios and merger orbits for a more robust prediction.

In this study, we did not focus on the 'tightness' of the local scaling relations of ETGs. Nipoti et al. (2009b) note that it is difficult to explain such an exact relation through the mass and size growth of compact massive ETGs at high $z$ with dry mergers. We will investigate the strength of the correlation during cosmological merger histories in future studies.

\section{ACKNOWLEDGMENTS}

We thank Volker Springel for making GADGET-2 publicly available. We thank M. Fujimoto, E. Tasker and C. Nipoti for useful suggestions. Numerical computations were carried out on Cray XT4 at the Center for Computational Astrophysics, CfCA, of the National Astronomical Observatory of Japan. The Millennium Simulation Data bases used in this paper and the web application providing online access to them were constructed as part of the activities of the German Astrophysical Virtual Observatory. This work was supported by Grant-in-Aid for Specially Promoted Research 20001003.

\section{REFERENCES}

Abadi M. G., Navarro J. F., Steinmetz M., 2006, MNRAS, 365, 747

Athanassoula E., 2005, inSzczerba R., Stasińska G., Gorny S. K., eds, AIP Conf. Ser. Vol. 804, Planetary Nebulae as Astronomical Tools, Melville, New York, pp. 333-340

Barkana R., Loeb A., 2001, Phys. Rep., 349, 125

Bell E. F. et al., 2006, ApJ, 640, 241

Bertin G., Ciotti L., Del Principe M., 2002, A\&A, 386, 149

Bezanson R., van Dokkum P. G., Tal T., Marchesini D., Kriek M., Franx M., Coppi P., 2009, ApJ, 697, 1290

Binney J., Merrifield M., 1998, in Binney J., Merrifield M., eds, Galactic astronomy. Princeton University Press, Princeton, NJ

Binney J., Tremaine S., 2008, inBinney J., Tremaine S., eds, Galactic Dynamics, 2nd edn. Princeton University Press, Princeton, NJ

Bolton A. S., Burles S., Koopmans L. V. E., Treu T., Gavazzi R., Moustakas L. A., Wayth R., Schlegel D. J., 2008, ApJ, 682, 964

Boylan-Kolchin M., Ma C.-P., 2007, MNRAS, 374, 1227

Boylan-Kolchin M., Ma C., Quataert E., 2005, MNRAS, 362, 184

Boylan-Kolchin M., Ma C., Quataert E., 2006, MNRAS, 369, 1081

Buitrago F., Trujillo I., Conselice C. J., Bouwens R. J., Dickinson M., Yan H., 2008, ApJ, 687, L61

Bullock J. S., Kolatt T. S., Sigad Y., Somerville R. S., Kravtsov A. V., Klypin A. A., Primack J. R., Dekel A., 2001, MNRAS, 321, 559

Bundy K., Fukugita M., Ellis R. S., Targett T. A., Belli S., Kodama T., 2009, ApJ, 697, 1369

Cappellari M. et al., 2009, ApJ, 704, L34

Cenarro A. J., Trujillo I., 2009, ApJ, 696, L43

Cimatti A. et al., 2008, A\&A, 482, 21

Ciotti L., 1996, ApJ, 471, 68

Ciotti L., Bertin G., 1999, A\&A, 352, 447

Ciotti L., Lanzoni B., Volonteri M., 2007, ApJ, 658, 65

Cox T. J., Dutta S. N., Di Matteo T., Hernquist L., Hopkins P. F., Robertson B., Springel V., 2006, ApJ, 650, 791
Croton D. J. et al., 2006, MNRAS, 365, 11

Damjanov I. et al., 2009, ApJ, 695, 101

De Lucia G., Blaizot J., 2007, MNRAS, 375, 2

Dressler A., 1980, ApJ, 236, 351

Dubinski J., Carlberg R. G., 1991, ApJ, 378, 496

El-Zant A., Shlosman I., Hoffman Y., 2001, ApJ, 560, 636

Fan L., Lapi A., De Zotti G., Danese L., 2008, ApJ, 689, L101

Graham A. W., Guzmán R., 2003, AJ, 125, 2936

Guo Q., White S. D. M., 2008, MNRAS, 384, 2

Hernquist L., 1990, ApJ, 356, 359

Hilz M., Naab T., Ostriker J. P., Thomas J., Burkert A., Jesseit R., 2012, arXiv e-print: 1206.1597

Hopkins P. F., Hernquist L., Cox T. J., Keres D., Wuyts S., 2009, ApJ, 691, 1424

Hopkins P. F. et al., 2010a, ApJ, 715, 202

Hopkins P. F., Bundy K., Hernquist L., Wuyts S., Cox T. J., 2010b, MNRAS, 401, 1099

Khochfar S., Silk J., 2006a, ApJ, 648, L21

Khochfar S., Silk J., 2006b, MNRAS, 370, 902

Kuijken K., Dubinski J., 1994, MNRAS, 269, 13

Lynden-Bell D., 1967, MNRAS, 136, 101

Ma C.-P., Boylan-Kolchin M., 2004, Phys. Rev. Lett., 93, 021301

Naab T., Johansson P. H., Ostriker J. P., 2009, ApJ, 699, L178

Navarro J. F., Frenk C. S., White S. D. M., 1997, ApJ, 490, 493

Newman A. B., Ellis R. S., Treu T., Bundy K., 2010, ApJ, 717, L103

Newman A. B., Ellis R. S., Bundy K., Treu T., 2012, ApJ, 746, 162

Nipoti C., Treu T., Bolton A. S., 2009a, ApJ, 703, 1531

Nipoti C., Treu T., Auger M. W., Bolton A. S., 2009b, ApJ, 706, L86

Nipoti C., Treu T., Leauthaud A., Bundy K., Newman A. B., Auger M. W., 2012, MNRAS, 422, 1714

Oser L., Naab T., Ostriker J. P., Johansson P. H., 2012, ApJ, 744, 63

Ragone-Figueroa C., Granato G. L., 2011, MNRAS, 414, 3690

Sersic J. L., 1968, Atlas de galaxias australes. Observatorio Astronomico, Cordoba, Argentina

Shankar F., Marulli F., Bernardi M., Mei S., Meert A., Vikram V., 2011, arXiv e-print: 1105.6043

Shen S., Mo H. J., White S. D. M., Blanton M. R., Kauffmann G., Voges W., Brinkmann J., Csabai I., 2003, MNRAS, 343, 978

Shih H.-Y., Stockton A., 2011, ApJ, 733, 45

Sommer-Larsen J., Toft S., 2010, ApJ, 721, 1755

Springel V., 2005, MNRAS, 364, 1105

Springel V., Di Matteo T., Hernquist L., 2005a, MNRAS, 361, 776

Springel V. et al., 2005b, Nat, 435, 629

Stewart K. R., Bullock J. S., Barton E. J., Wechsler R. H., 2009, ApJ, 702, 1005

Stockton A., Shih H.-Y., Larson K., 2010, ApJ, 709, L58

Taylor E. N., Franx M., Glazebrook K., Brinchmann J., van der Wel A., van Dokkum P. G., 2010, ApJ, 720, 723

Thomas D., Maraston C., Bender R., Mendes de Oliveira C., 2005, ApJ, 621,673

Trujillo I. et al., 2006, ApJ, 650, 18

Trujillo I., Conselice C. J., Bundy K., Cooper M. C., Eisenhardt P., Ellis R. S., 2007, MNRAS, 382, 109

Trujillo I., Carrasco E. R., Ferre-Mateu A., 2012, ApJ, 751, 45

Valentinuzzi T. et al., 2010, ApJ, 712, 226

van de Sande J. et al., 2011, ApJ, 736, L9

van Dokkum P. G. et al., 2008, ApJ, 677, L5

van Dokkum P. G., Kriek M., Franx M., 2009, Nat, 460, 717

van Dokkum P. G. et al., 2010, ApJ, 709, 1018

This paper has been typeset from a $\mathrm{T}_{\mathrm{E}} \mathrm{X} / \mathrm{LAT} \mathrm{T}$ file prepared by the author. 